\title{
INFLUENCE OF COLD REDUCTION AND HEAT TREATMENT \\ ON THE PROPERTIES AND MICROSTRUCTURE \\ OF ALLOY 718 FASTENER STOCK
}

\author{
R. A. Heacox
}

Latrobe Stee1 Company

Latrobe, Pennsylvania

\begin{abstract}
Recent1y, Alloy 718 has come into use as a cold reduced and aged alloy for high strength fastener applications. This investigation was undertaken to define the property levels as they relate to (1) amount of cold reduction, (2) aging treatment, (3) prior solution treatment, and (4) bar size.

bars were solution treated at $1750 \mathrm{~F}$ or $1840 \mathrm{~F}$ prior to cold drawing 20 to 50 percent to two (.875" and .375" round) bar sizes. Specimens from each bar were then given the conventional $1325 \mathrm{~F}+1150 \mathrm{~F}$ age or one of two modified aging cycles. One modified aging cycle, $1275 \mathrm{~F}+1150 \mathrm{~F}$, was designed to produce optimum tensile strength; the other, $1450 \mathrm{~F}+$ $1150 \mathrm{~F}$, was designed to produce optimum stress-rupture properties. Light and electron metallographic studies were conducted to correlate structure with properties.
\end{abstract}

The results obtained in this investigation indicated that:

(1) Tensile and yield strengths and rupture ductility increase with increasing cold reduction; whereas, tensile ductility and rupture strength decrease.

(2) Aging treatment has a significant effect on properties. The $1275 \mathrm{~F}+1150 \mathrm{~F}$ cycle produces the best tensile and yield strengths. Optimum rupture properties result from use of the $1450 \mathrm{~F}+1150 \mathrm{~F}$ cycle. The best balance of mechanical properties, however, is obtained by using the conventional $1325 \mathrm{Ft}$ $1150 \mathrm{~F}$ cycle. The conventional age $(1325 \mathrm{~F}+1150 \mathrm{~F})$ and one modified age $(1450 \mathrm{~F}+$ $1150 \mathrm{~F})$ results in overaging at the higher cold reductions $(40-50 \%)$.

(3) Solution heat treating temperature does not have a significant effect on mechanical properties.

(4) The .375" round bars exhibit higher tensile and yield strengths and rupture ductilities than the $.875^{\prime \prime}$ round bars.

\section{INTRODUCTION}

Alloy 718 is a wrought nickel-base alloy developed by H. L. Eiselstein, International Nickel Company, for moderately high temperature applications $(1)$. Currently, the alloy is being utilized in both cryogenic and elevated-temperature applications in the form of sheet, bar and forgings.

In the last several years, Alloy 718 has come into use as a cold drawn and aged alloy for high strength fastener applications. This application resulted from the ability of Alloy 718, after minimal cold drawing and aging, to attain strengths in excess of $200 \mathrm{ksi}$ with sufficient ductility and to retain the strength increase achieved by cold reduction (and aging) to temperatures in excess of $1200 \mathrm{~F}$.

This investigation was undertaken to define the property levels as they relate to: 
(1) amount of cold reduction, (2) aging treatment, (3) bar size, and (4) pre-draw solution treatment. Light and electron metallographic studies were conducted to determine the effect of the experimental variables on microstructure and to correlate microstructure with properties.

\section{EXPERIMENTAL PROCEDURE}

For this investigation, an 8" round billet was obtained from production stock. The chemistry of this double vacuum melted product is listed in Table 1.

The billet was cogged and hot rolled to $1.250^{\prime \prime}$ round and .550" round bars using standard production procedures. Eight bars of each size were then processed as shown in Table 2 to $.875^{\prime \prime}$ round and $.375^{\prime \prime}$ round cold drawn bars. The hot rolled bars were ground to different start sizes in order to cold draw to two common sizes. Reductions of 30 percent and less were done in one cold draw pass and those over 30 percent were achieved in two passes.

Ten specimen blanks, identified 1 through 10, were cut from each bar and precipitation heat treated as follows:

Specimen 1 - none (as cold reduced)

Specimens 2,3 and $4-1325 \mathrm{~F} / 8 \mathrm{Hr} / \mathrm{FC} 100 \mathrm{~F} / \mathrm{Hr}$ to $1150 \mathrm{~F} / 8 \mathrm{Hr} / \mathrm{AC}$

Specimens 5,6 and $7-1275 \mathrm{~F} / 3 \mathrm{Hr} / \mathrm{FC} 100 \mathrm{~F} / \mathrm{Hr}$ to $1150 \mathrm{~F} / 8 \mathrm{Hr} / \mathrm{AC}$

Specimens 8,9 and $10-1450 \mathrm{~F} / 4 \mathrm{Hr} / \mathrm{WQ}+1150 \mathrm{~F} / 8 \mathrm{Hr} / \mathrm{AC}$

The $1325 \mathrm{~F}+1150 \mathrm{~F}$ age is the conventional A1loy 718 aging treatment. The $1275 \mathrm{~F}+1150 \mathrm{~F}$ age was selected to obtain optimum tensile strengths; whereas, the $1450 \mathrm{~F}+1150 \mathrm{~F}$ was designed to produce optimum rupture ductility without too great a sacrifice in strength properties. The modified aging treatments were selected on the basis of (1) hardness
data from gradient bar studies, and (2) from stress rupture tests conducted previously

The blanks were crush-ground into conventional ASTM .252" diameter tensile specimens and ASTM .178" diameter smooth bar stress rupture specimens. The specimens were tested as follows:

Specimens $1,2,5$ and 8 - room temperature tensiles

Specimens 3,6 and 9 - 1000F tensile

Specimens 4,7 and 10 - 1000F stress rupture - Stress was variable and was determined as 95 percent of the 1000F yield strength of each specimen.

Elevated temperature testing was done at $1000 \mathrm{~F}$ because this temperature is of more significance for fastener applications than conventional $1200 \mathrm{~F}$ testing. The variable rupture stress was used to provide the same stress-yield strength parameter for a 11 specimens. (A constant stress could not be used because of the large variations in strengths obtained through cold reduction and aging.)

Metallographic samples were obtained from each test specimen for optical and electron microscopic examinations. Conventional metallographic preparation techniques were used. For electron microscopy, collodion replicas were shadowed with chromium and backed by a thin layer of carbon. 
Table 1

Chemistry of Alloy 718 Billet

$\begin{array}{ccccccccccccccc}\underline{C} & \underline{M n} & \underline{\mathrm{Si}} & \underline{\mathrm{S}} & \underline{\mathrm{P}} & \underline{\mathrm{Cr}} & \underline{\mathrm{Fe}} & \underline{\mathrm{Co}} & \underline{\text { Mo }} & \underline{\mathrm{Cb}+\mathrm{Ta}} & \underline{\mathrm{Ti}} & \underline{\mathrm{A}} & \underline{\mathrm{B}} & \underline{\mathrm{Cu}} & \underline{\mathrm{Ni}} \\ .04 & .02 & .05 & .003 & .005 & 18.91 & 19.40 & .11 & 3.00 & 5.11 & 1.03 & .56 & .005 & .03 & \mathrm{Ba1}\end{array}$

Table 2

Processing of Bars

Identity

Solution Heat Treat

$1750 \mathrm{~F} / 1 \mathrm{hr} / \mathrm{WQ}$

$1840 \mathrm{~F} / 1 \mathrm{hr} / \mathrm{WQ}$

Centerless Grind

Cold Draw
Hot Roll Size

$\begin{array}{ll}\frac{1.250^{\prime \prime} \mathrm{Rd}}{\text { Thru } 8} & \frac{.550^{\prime \prime} \mathrm{Rd}}{\bar{A} \text { thru H }} \\ & \\ 7 \text { thru } 4 & \text { A thru D } \\ 5 \text { thru } 8 & \text { E thru H }\end{array}$

$A$ and $E$ to .420"

$B$ and $F$ to .448"

$C$ and $G$ to $.484^{\prime \prime}$

$D$ and $H$ to $.530^{\prime \prime}$

1 and $520 \%$ to . $875^{\prime \prime}$

2 and $630 \%$ to . $875^{\prime \prime}$

$A$ and $E 20 \%$ to $.375^{\prime \prime}$

$B$ and $F 30 \%$ to $.375^{\prime \prime}$

$C$ and $G 40 \%$ to $.375^{\prime \prime}$

$D$ and $H 50 \%$ to $.375^{\prime \prime}$ 


\section{RESULTS AND DISCUSSION}

\section{Mechanical Properties}

At present, fastener specifications for high strength Alloy 718 require only roomtemperature tensile testing. For this reason, and because the 1000F tensile data follow the same trends as the room-temperature data, the figures (and discussion) presented in this section consider only the room-temperature tensile data. Because a11 the properties, regardless of bar size and prior solution temperature, exhibit the same trend with increasing amounts of cold reduction, only the data from the .875" round bars given a prior $1750 \mathrm{~F}$ solution treatment are plotted in graphical form (except where necessary to show the effect of bar size and solutioning temperature). All data are given in table form.

\section{Effect of Cold Reduction}

As Cold Reduced. The room temperature tensile properties, hardnesses and grain sizes of the bars in the cold reduced condition are presented in Table 3 . The selected tensile and yield strength and elongation data (.875" RD.-1750F SHT) are shown graphically in Figure 1.

For a given solution treatment and size, the tensile strength varies on the average of about $60 \mathrm{ksi}$ over the range of cold reductions studied. Variations in yield strength are even greater; the yield strength of the $.375^{\prime \prime}$ bar solution treated at $1840 \mathrm{~F}$ varies from $126 \mathrm{ksi}$ at 20 percent cold work to $207 \mathrm{ksi}$ after 50 percent reduction. Elongation values decreased rapidly above 20 percent cold work dropping from as high as 25.5 percent at 20 percent cold work to 13.5 percent at 30 percent reduction. Above 30 percent reduction the decrease becomes less. Although the reduction of area values show a constant decrease with increasing amounts of cold work, the minimum value obtained (24.8\%) is sufficiently high so that no problems would be encountered with this property.

Rockwell $C$ hardness increases with increasing cold work with the highest hardnesses being obtained with the lower pre-draw solution temperature and the smaller bar size. Likewise, the finest grain sizes were produced in the . $375^{\prime \prime}$ bar given the 1750F solution treatment.

Conventiona 1 Age. Property data for specimens given the conventional $1325 \mathrm{~F}+1150 \mathrm{~F}$ age are presented in Tables 4 and 5 for the $.875^{\prime \prime}$ and $.375^{\prime \prime}$ round bars, respectively. A plot of the tensile and yield strength and tensile elongation data for the .875" bar given a prior 1750F solution is shown in Figure 2. These data show trends, with increasing cold reduction, that are similar to those exhibited by the cold reduced bars. An increase in cold work from 20 to 50 percent results in an increase of up to $50 \mathrm{ksi}$ in tensile strength and $30 \mathrm{ksi}$ in yield strength. Note that the yield strength shows a tendency to level off at 50 percent reduction.

If one considers that the tensile and yield strengths of hot rolled and conventionally heat treated Alloy 718 small bars are typically 200 and $170 \mathrm{ksi}$, respectively; then an increase of about $70 \mathrm{ksi}$ in both tensile and yield strength can be achieved by cold working 50 percent prior to aging.

Rupture strength decreases rapidly with increasing cold reduction; whereas, rupture elongation shows a tendency to increase, Figure 3 . This behavior is believed due to stress-induced aging effects during stress rupture testing at 1000F. For a given size or solution treatment the aging reactions proceed more rapidly as the amount of cold work increases. 
Table 3

Mechanical Properties after Cold Reduction

\begin{tabular}{|c|c|c|c|c|c|c|c|c|c|}
\hline $\begin{array}{l}\text { BAR } \\
\text { SIZE } \\
\end{array}$ & $\begin{array}{l}\text { SOLUTION } \\
\text { TEMP. } \\
F \\
\end{array}$ & $\begin{array}{c}\text { NOMINAL } \\
\text { COLD } \\
\text { REDUCTION } \\
\% \\
\end{array}$ & $\begin{array}{l}\text { SPECIMEN } \\
\text { IDENTITY } \\
\end{array}$ & $\begin{array}{l}\text { UTS } \\
\text { ksi }\end{array}$ & $\begin{array}{l}.2 Y S \\
\mathrm{ksi} \\
\end{array}$ & $\begin{array}{c}\text { ELONG. } \\
\% \\
\end{array}$ & $\begin{array}{l}\text { R.A. } \\
\% \\
\end{array}$ & $\underline{R_{C}}$ & $\begin{array}{c}\text { ASTM } \\
\text { GRAIN SIZE }\end{array}$ \\
\hline $.875^{\prime \prime}$ & 1750 & $\begin{array}{l}20 \\
30 \\
40 \\
50 \\
20 \\
30 \\
40 \\
50\end{array}$ & $\begin{array}{l}1-1 \\
2-1 \\
3-1 \\
4-1 \\
5-1 \\
6-1 \\
7-1 \\
8-1\end{array}$ & $\begin{array}{l}155.5 \\
183.6 \\
205.5 \\
224.0 \\
149.1 \\
179.6 \\
196.4 \\
214.9\end{array}$ & $\begin{array}{l}132.3 \\
179.4 \\
195.4 \\
200.4 \\
134.3 \\
179.6 \\
196.4 \\
207.2\end{array}$ & $\begin{array}{r}25.0 \\
13.5 \\
9.0 \\
10.0 \\
25.5 \\
13.5 \\
8.5 \\
9.0\end{array}$ & $\begin{array}{l}47.9 \\
44.3 \\
42.5 \\
33.9 \\
52.9 \\
45.1 \\
41.3 \\
38.0\end{array}$ & $\begin{array}{l}32.0 \\
36.5 \\
40.5 \\
42.0 \\
32.0 \\
34.5 \\
38.0 \\
42.0\end{array}$ & $6 / 7$ some 4 \\
\hline $.375^{\prime \prime}$ & 1750 & $\begin{array}{l}20 \\
30 \\
40 \\
50\end{array}$ & $\begin{array}{l}A-1 \\
B-1 \\
C-1 \\
D-1\end{array}$ & $\begin{array}{l}174.7 \\
195.2 \\
215.0 \\
229.5\end{array}$ & $\begin{array}{l}151.3 \\
184.4 \\
205.6 \\
210.5\end{array}$ & $\begin{array}{r}12.5 \\
10.5 \\
9.3 \\
\text { Broke } \\
\text { Threa }\end{array}$ & $\begin{array}{l}38.9 \\
33.9 \\
24.8 \\
\text { in } \\
\text { ads }\end{array}$ & $\begin{array}{l}37.3 \\
39.0 \\
42.5 \\
43.3\end{array}$ & $8+$ \\
\hline & 1840 & $\begin{array}{l}20 \\
30 \\
40 \\
50\end{array}$ & $\begin{array}{l}E-1 \\
F-1 \\
G-1 \\
H-1\end{array}$ & $\begin{array}{l}149.3 \\
174.1 \\
196.8 \\
212.1\end{array}$ & $\begin{array}{l}125.8 \\
167.7 \\
192.4 \\
206.5\end{array}$ & $\begin{array}{r}22.0 \\
14.0 \\
10.0 \\
9.3\end{array}$ & $\begin{array}{l}55.9 \\
47.5 \\
41.3 \\
42.9\end{array}$ & $\begin{array}{l}30.6 \\
35.7 \\
37.7 \\
41.0\end{array}$ & $5 / 7$ \\
\hline
\end{tabular}


Table 4

Mechanical Properties after $1325 \mathrm{~F} / 8 \mathrm{hr} / \mathrm{FC} 100 \mathrm{~F} / \mathrm{hr}$ to $1150 \mathrm{~F} / 8 \mathrm{hr} / \mathrm{AC}$ $.875^{\prime \prime}$ Round

\begin{tabular}{|c|c|c|c|c|c|c|c|c|c|}
\hline $\begin{array}{c}\text { SOLUTION } \\
\text { TEMP. } \\
\text { F } \\
\end{array}$ & $\begin{array}{c}\text { NOMINAL } \\
\text { COLD } \\
\text { REDUCTION } \\
\% \\
\end{array}$ & $\begin{array}{l}\text { SPECIMEN } \\
\text { IDENTITY } \\
\end{array}$ & $\begin{array}{c}\text { TEST } \\
\text { CONDITIONS } \\
\end{array}$ & $\begin{array}{l}\text { UTS } \\
\text { ksi }\end{array}$ & $\begin{array}{l}.2 Y S \\
\mathrm{ksi} \\
\end{array}$ & $\begin{array}{c}\text { ELONG. } \\
\%\end{array}$ & $\begin{array}{l}\text { R.A. } \\
\%\end{array}$ & $\begin{array}{c}\text { STRESS } \\
\text { RUPTURE } \\
\text { LIFE } \\
\mathrm{hr} \\
\end{array}$ & $\underline{R_{C}}$ \\
\hline \multirow[t]{4}{*}{1750} & 20 & $\begin{array}{l}1-2 \\
1-3 \\
1-4\end{array}$ & $\begin{array}{l}\mathrm{RT} \\
1000 \mathrm{~F} \\
1000 \mathrm{~F} / \\
165 \mathrm{ks} \mathrm{i}\end{array}$ & $\begin{array}{l}220.0 \\
191.6\end{array}$ & $\begin{array}{l}198.4 \\
173.7\end{array}$ & $\begin{array}{l}17.0 \\
15.5 \\
11.5\end{array}$ & $\begin{array}{l}38.3 \\
37.3 \\
16.4\end{array}$ & 823.9 & 46.7 \\
\hline & 30 & $\begin{array}{l}2-2 \\
2-3 \\
2-4\end{array}$ & $\begin{array}{r}R T \\
1000 \mathrm{~F} \\
1000 \mathrm{~F} / \\
185 \mathrm{ks}\end{array}$ & $\begin{array}{l}236.7 \\
209.2\end{array}$ & $\begin{array}{l}223.6 \\
194.4\end{array}$ & $\begin{array}{l}11.5 \\
10.0 \\
12.9\end{array}$ & $\begin{array}{l}30.9 \\
31.9 \\
28.9\end{array}$ & 145.6 & 47.5 \\
\hline & 40 & $\begin{array}{l}3-2 \\
3-3 \\
3-4\end{array}$ & $\begin{array}{c}\mathrm{RT} \\
1000 \mathrm{~F} \\
1000 \mathrm{~F} / \\
194 \mathrm{ks} i\end{array}$ & $\begin{array}{l}253.7 \\
219.6\end{array}$ & $\begin{array}{l}228.5 \\
218.4\end{array}$ & $\begin{array}{r}9.5 \\
7.0 \\
14.3\end{array}$ & $\begin{array}{l}23.8 \\
23.4 \\
15.9\end{array}$ & 75.2 & 49.8 \\
\hline & 50 & $\begin{array}{l}4-2 \\
4-3 \\
4-4\end{array}$ & $\begin{array}{r}\mathrm{RT} \\
1000 \mathrm{~F} \\
1000 \mathrm{~F} / \\
207 \mathrm{ks} \mathrm{i}\end{array}$ & $\begin{array}{l}268.5 \\
232.5\end{array}$ & $\begin{array}{l}228.5 \\
218.4\end{array}$ & $\begin{array}{r}7.0 \\
7.5 \\
15.7\end{array}$ & $\begin{array}{l}19.6 \\
24.4 \\
27.1\end{array}$ & 20.8 & 49.0 \\
\hline \multirow[t]{4}{*}{1840} & 20 & $\begin{array}{l}5-2 \\
5-3 \\
5-4\end{array}$ & $\begin{array}{r}\mathrm{RT} \\
1000 \mathrm{~F} \\
1000 \mathrm{~F} / \\
163 \mathrm{ksi}\end{array}$ & $\begin{array}{l}218.8 \\
193.2\end{array}$ & $\begin{array}{l}198.6 \\
172.0\end{array}$ & $\begin{array}{r}18.0 \\
14.0 \\
8.6\end{array}$ & $\begin{array}{l}37.9 \\
34.4 \\
10.7\end{array}$ & 909.9 & 45.5 \\
\hline & 30 & $\begin{array}{l}6-2 \\
6-3 \\
6-4\end{array}$ & $\begin{array}{c}\text { RT } \\
\text { 1000F } \\
1000 \mathrm{~F} / \\
190 \mathrm{ksi}\end{array}$ & $\begin{array}{l}233.9 \\
213.2\end{array}$ & $\begin{array}{l}227.5 \\
200.4\end{array}$ & $\begin{array}{r}12.0 \\
11.0 \\
5.7\end{array}$ & $\begin{array}{l}30.9 \\
29.3 \\
13.3\end{array}$ & 45.0 & 47.3 \\
\hline & 40 & $\begin{array}{l}7-2 \\
7-3 \\
7-4\end{array}$ & $\begin{array}{r}\mathrm{RT} \\
1000 \mathrm{~F} \\
1000 \mathrm{~F} / \\
196 \mathrm{ksi}\end{array}$ & $\begin{array}{l}244.9 \\
276.6\end{array}$ & $\begin{array}{l}228.5 \\
206.1\end{array}$ & $\begin{array}{r}10.0 \\
8.0 \\
14.3\end{array}$ & $\begin{array}{l}27.9 \\
26.1 \\
23.3\end{array}$ & 96.2 & 48.5 \\
\hline & 50 & $\begin{array}{l}8-2 \\
8-3 \\
8-4\end{array}$ & $\begin{array}{c}\mathrm{RT} \\
1000 \mathrm{~F} \\
1000 \mathrm{~F} / \\
200 \mathrm{ksi}\end{array}$ & $\begin{array}{l}261.3 \\
226.2\end{array}$ & $\begin{array}{l}228.5 \\
210.7\end{array}$ & $\begin{array}{r}7.0 \\
8.0 \\
10.0\end{array}$ & $\begin{array}{l}19.8 \\
16.7 \\
25.1\end{array}$ & 43.5 & 49.0 \\
\hline
\end{tabular}


Table 5

Mechanical Properties after $1325 \mathrm{~F} / 8 \mathrm{hr} / \mathrm{FC} 100 \mathrm{~F} / \mathrm{hr}$ to $1150 \mathrm{~F} / 8 \mathrm{hr} / \mathrm{AC}$ $.375^{\prime \prime}$ Round

\begin{tabular}{|c|c|c|c|c|c|c|c|c|c|}
\hline $\begin{array}{l}\text { SOLUTION } \\
\text { TEMP. } \\
\mathrm{F} \\
\end{array}$ & $\begin{array}{c}\text { NOMINAL } \\
\text { COLD } \\
\text { REDUCTION } \\
\% \\
\end{array}$ & $\begin{array}{l}\text { SPECIMEN } \\
\text { IDENTITY } \\
\end{array}$ & $\begin{array}{c}\text { TEST } \\
\text { CONDITIONS } \\
\end{array}$ & $\begin{array}{l}\text { UTS } \\
\text { ks i } \\
\end{array}$ & $\begin{array}{l}.2 Y S \\
\mathrm{ksi} \\
\end{array}$ & $\begin{array}{c}\text { ELONG. } \\
\%\end{array}$ & $\begin{array}{l}\mathrm{R} . \mathrm{A} . \\
\% \\
\end{array}$ & $\begin{array}{c}\text { STRESS } \\
\text { RUPTURE } \\
\text { LIFE } \\
h r \\
\end{array}$ & $\underline{R_{C}}$ \\
\hline \multirow[t]{4}{*}{1750} & 20 & $\begin{array}{l}A-2 \\
A-3 \\
A-4\end{array}$ & $\begin{array}{c}\mathrm{RT} \\
1000 \mathrm{~F} \\
1000 \mathrm{~F} / \\
174 \mathrm{ksi}\end{array}$ & $\begin{array}{l}236.2 \\
208.0\end{array}$ & $\begin{array}{l}215.3 \\
182.7\end{array}$ & $\begin{array}{l}22.0 \\
11.4 \\
15.7\end{array}$ & $\begin{array}{l}28.1 \\
30.1 \\
30.3\end{array}$ & 15.5 & 48.7 \\
\hline & 30 & $\begin{array}{l}B-2 \\
B-3 \\
B-4\end{array}$ & $\begin{array}{c}\mathrm{RT} \\
1000 \mathrm{~F} \\
1000 \mathrm{~F} / \\
188 \mathrm{ks} \mathrm{i}\end{array}$ & $\begin{array}{l}247.5 \\
221.2\end{array}$ & $\begin{array}{l}225.4 \\
198.4\end{array}$ & $\begin{array}{r}9.5 \\
10.0 \\
15.7\end{array}$ & $\begin{array}{l}26.3 \\
29.3 \\
30.1\end{array}$ & 13.5 & 48.7 \\
\hline & 40 & $\begin{array}{l}C-2 \\
C-3 \\
C-4\end{array}$ & $\begin{array}{c}\mathrm{RT} \\
1000 \mathrm{~F} \\
1000 \mathrm{~F} / \\
194 \mathrm{ksi}\end{array}$ & $\begin{array}{l}260.4 \\
223.2\end{array}$ & $\begin{array}{l}242.9 \\
204.0\end{array}$ & $\begin{array}{r}8.6 \\
7.9 \\
15.7\end{array}$ & $\begin{array}{l}15.0 \\
25.6 \\
29.5\end{array}$ & 17.3 & 48.5 \\
\hline & 50 & $\begin{array}{l}D-2 \\
D-3 \\
D-4\end{array}$ & $\begin{array}{c}\mathrm{RT} \\
1000 \mathrm{~F} \\
1000 \mathrm{~F} / \\
200 \mathrm{ksi}\end{array}$ & $\begin{array}{l}272.3 \\
229.4\end{array}$ & $\begin{array}{l}236.9 \\
210.2\end{array}$ & $\begin{array}{r}7.1 \\
8.6 \\
15.7\end{array}$ & $\begin{array}{l}10.0 \\
24.1 \\
29.0\end{array}$ & 23.0 & 49.7 \\
\hline \multirow[t]{4}{*}{1840} & 20 & $\begin{array}{l}E-2 \\
E-3 \\
E-4\end{array}$ & $\begin{array}{c}\mathrm{RT} \\
1000 \mathrm{~F} \\
1000 \mathrm{~F} / \\
162 \mathrm{ksi}\end{array}$ & $\begin{array}{l}217.6 \\
191.2\end{array}$ & $\begin{array}{l}202.4 \\
170.3\end{array}$ & $\begin{array}{r}17.0 \\
12.0 \\
5.7\end{array}$ & $\begin{array}{l}41.2 \\
38.9 \\
11.6\end{array}$ & 318.7 & 42.8 \\
\hline & 30 & $\begin{array}{l}F-2 \\
F-3 \\
F-4\end{array}$ & $\begin{array}{c}\mathrm{RT} \\
1000 \mathrm{~F} \\
1000 \mathrm{~F} / \\
183 \mathrm{ksi}\end{array}$ & $\begin{array}{l}235.1 \\
208.0\end{array}$ & $\begin{array}{l}279.6 \\
192.4\end{array}$ & $\begin{array}{r}12.0 \\
17.0 \\
5.7\end{array}$ & $\begin{array}{l}35.3 \\
33.9 \\
12.4\end{array}$ & 66.6 & 47.7 \\
\hline & 40 & $\begin{array}{l}G-2 \\
G-3 \\
G-4\end{array}$ & $\begin{array}{c}\text { RT } \\
1000 \mathrm{~F} \\
1000 \mathrm{~F} / \\
196 \mathrm{ksi}\end{array}$ & $\begin{array}{l}250.0 \\
279.6\end{array}$ & $\begin{array}{l}228.5 \\
206.4\end{array}$ & $\begin{array}{r}10.0 \\
8.0 \\
10.0\end{array}$ & $\begin{array}{l}27.3 \\
27.3 \\
24.8\end{array}$ & 42.0 & 48.5 \\
\hline & 50 & $\begin{array}{l}H-2 \\
H-3 \\
H-4\end{array}$ & $\begin{array}{c}\text { RT } \\
1000 \mathrm{~F} \\
1000 \mathrm{FI} \\
202 \mathrm{ksi}\end{array}$ & $\begin{array}{l}262.0 \\
230.5\end{array}$ & $\begin{array}{l}244.0 \\
212.9\end{array}$ & $\begin{array}{r}7.9 \\
7.9 \\
14.3\end{array}$ & $\begin{array}{l}20.0 \\
25.3 \\
29.8\end{array}$ & 41.2 & 49.1 \\
\hline
\end{tabular}


High-Strength Age. The mechanical properties of specimens after the $1275 \mathrm{~F}+1150 \mathrm{~F}$ high-strength age are given Tables 6 and 7 . The tensile and yield strength and tensile elongation data for the $.875^{\prime \prime}$ barsgiven the 1750F solution treatment are presented graphically in Figure 4. Once again these data show the same trends with increasing amounts of cold work as did those plotted in Figures 1 and 2. One will note, however, that after the $1275 \mathrm{~F}+1150 \mathrm{~F}$ age, yield strength does not have the same tendency to level off at the 50 percent cold reduction level. After the high-strength age, tensile strength increases about $60 \mathrm{ksi}$ and yield strength about $70 \mathrm{ksi}$ with increasing cold reduction from 20 to 50 percent.

Figure 5 indicates that rupture properties, although incomplete, show the same trends with increasing cold work as did those for the conventional age, that is, life decreases and ductility increases with increasing cold reduction.

Stress-Rupture Age. Mechanical property data for the $1450 \mathrm{~F} / 4 \mathrm{Hr} / \mathrm{WQ}+1150 \mathrm{~F} / 8 \mathrm{Hr} / \mathrm{AC}$ age are listed in Tables 8 and 9. A plot of the room temperature tensile data (.875" RD, $1750 \mathrm{~F}$ SHT) is shown in Figure 6 . This age results in only a modest increase in strength over the range of cold reduction studied. As for the conventional age, the yield strength tends to level off at the 50 percent cold reduction level. Tensile ductilities again decrease with increasing cold work.

Unfortunately, the stress rupture properties for this age are incomplete. However, preliminary data, Figure 7, indicate that life and elongation exhibit the same trends toward decreased life and increased elongation with increasing cold reduction.

\section{Effect of Aging Cycle}

Aging cycle had a significant effect on tensile properties as shown in Figures 8,9 and 10 which show tensile strength, yield strength, and tensile elongation as a function of aging treatment, respectively. Figures 8 and 9 show that, except for the 20 percent reduction level, the $1275 \mathrm{~F}+1150 \mathrm{~F}$ age results in the highest tensile and yield strengths.

The tendency of the yield strength to drop off drastically at 50 percent reduction and to a lesser extent at 40 percent reduction after aging at $1325 \mathrm{~F}+1150 \mathrm{~F}$ or $1450 \mathrm{~F}+$ 1150F, Figure 9, suggests that these aging cycles result in overaging at the higher reduction levels. As indicated by Figure 10, tensile elongation increases as the initial aging temperature increases.

Figure 11 shows a plot of tensile strength vs. tensile elongation for the .875" specimen given a prior $1750 \mathrm{~F}$ solution. These curves indicate that the $1325 \mathrm{~F}+1150 \mathrm{~F}$ aging cycle results in the best combination of tensile strength and ductility.

A plot of a11 the tensile strength and tersile elongation data is presented in Figure 12. These data show that tensile strengths on the order of $250 \mathrm{ksi}$ can be achieved with tensile elongations of 7 to 12 percent.

Although the stress rupture data are incomplete, preliminary data indicate that the $1450 \mathrm{~F}+1150 \mathrm{~F}$ age results in the best rupture properties. In addition, the available data show that for a given reduction level, rupture strength increases as the initial aying temperature increases.

\section{Effect of Solution Heat Treatment}

The effect of solution heat treatment on the tensile strength and elongation of .875" specimens given the conventional $1325 \mathrm{~F}+1150 \mathrm{~F}$ age is shown in Figure 13. Although 
Table 6

Mechanical Properties after $1275 \mathrm{~F} / 8 \mathrm{hr} / \mathrm{FC} 100 \mathrm{~F} / \mathrm{hr}$ to $1150 \mathrm{~F} / 8 \mathrm{hr} / \mathrm{AC}$ $.875^{\text {II Round }}$

\begin{tabular}{|c|c|c|c|c|c|c|c|c|c|}
\hline $\begin{array}{c}\text { SOLUTION } \\
\text { TEMP. } \\
F \\
\end{array}$ & $\begin{array}{c}\text { NOMINAL } \\
\text { COLD } \\
\text { REDUCTION } \\
\% \\
\end{array}$ & $\begin{array}{l}\text { SPECIMEN } \\
\text { IDENTITY } \\
\end{array}$ & $\begin{array}{c}\text { TEST } \\
\text { CONDITIONS } \\
\end{array}$ & $\begin{array}{l}\text { UTS } \\
\text { ksi } \\
\end{array}$ & $\begin{array}{l}.2 Y S \\
\mathrm{ksi} \\
\end{array}$ & $\begin{array}{c}\text { ELONG. } \\
\%\end{array}$ & $\begin{array}{c}\text { R.A. } \\
\% \\
\end{array}$ & $\begin{array}{c}\text { STRESS } \\
\text { RUPTURE } \\
\text { LIFE } \\
\text { hr } \\
\end{array}$ & $\underline{R_{C}}$ \\
\hline \multirow[t]{4}{*}{1750} & 20 & $\begin{array}{l}1-5 \\
1-6 \\
1-7\end{array}$ & $\begin{array}{r}\mathrm{RT} \\
1000 \mathrm{~F} \\
1000 \mathrm{~F} / \\
160 \mathrm{ksi}\end{array}$ & $\begin{array}{l}216.6 \\
185.1\end{array}$ & $\begin{array}{l}196.4 \\
168.5\end{array}$ & \multicolumn{2}{|c|}{ (In test) } & & 45.7 \\
\hline & 30 & $\begin{array}{l}2-5 \\
2-6 \\
2-7\end{array}$ & $\begin{array}{c}\mathrm{RT} \\
1000 \mathrm{~F} \\
1000 \mathrm{~F} / \\
187 \mathrm{ks}\end{array}$ & $\begin{array}{l}238.9 \\
205.2\end{array}$ & $\begin{array}{l}234.5 \\
197.2\end{array}$ & $\begin{array}{r}10.5 \\
8.5 \\
14.3\end{array}$ & $\begin{array}{r}35.1 \\
34.2 \\
8.8\end{array}$ & 448.6 & 48.3 \\
\hline & 40 & $\begin{array}{l}3-5 \\
3-6 \\
3-7\end{array}$ & $\begin{array}{c}\mathrm{RT} \\
1000 \mathrm{~F} \\
1000 \mathrm{~F} / \\
199 \mathrm{ksi}\end{array}$ & $\begin{array}{l}271.5 \\
218.1\end{array}$ & $\begin{array}{l}251.5 \\
209.3\end{array}$ & $\begin{array}{l}6.0 \\
7.0 \\
8.6\end{array}$ & $\begin{array}{r}22.7 \\
21.3 \\
7.2\end{array}$ & 179.8 & 48.3 \\
\hline & 50 & $\begin{array}{l}4-5 \\
4-6 \\
4-7\end{array}$ & $\begin{array}{c}\mathrm{RT} \\
1000 \mathrm{~F} \\
1000 \mathrm{~F} / \\
279 \mathrm{ksi}\end{array}$ & $\begin{array}{l}281.0 \\
235.5\end{array}$ & $\begin{array}{l}277.4 \\
230.5\end{array}$ & $\begin{array}{r}5.0 \\
5.5 \\
10.0\end{array}$ & $\begin{array}{l}18.6 \\
21.0 \\
26.8\end{array}$ & 32.4 & 49.4 \\
\hline \multirow[t]{4}{*}{1840} & 20 & $\begin{array}{l}5-5 \\
5-6 \\
5-7\end{array}$ & $\begin{array}{c}\mathrm{RT} \\
1000 \mathrm{~F} \\
1000 \mathrm{~F} / \\
163 \mathrm{ks} \mathrm{i}\end{array}$ & $\begin{array}{l}213.6 \\
183.8\end{array}$ & $\begin{array}{l}196.4 \\
171.7\end{array}$ & $\begin{array}{l}15.0 \\
15.0\end{array}$ & \multicolumn{2}{|c|}{$\begin{array}{c}43.7 \\
40.2 \\
\text { (In test) }\end{array}$} & 46.1 \\
\hline & 30 & $\begin{array}{l}6-5 \\
6-6 \\
6-7\end{array}$ & $\begin{array}{c}\mathrm{RT} \\
1000 \mathrm{~F} \\
1000 \mathrm{~F} / \\
186 \mathrm{ks} i\end{array}$ & $\begin{array}{l}239.7 \\
202.8\end{array}$ & $\begin{array}{l}236.5 \\
196.1\end{array}$ & $\begin{array}{r}10.5 \\
8.0 \\
2.9\end{array}$ & \multicolumn{2}{|l|}{$\begin{array}{r}34.5 \\
32.1 \\
4.8\end{array}$} & 46.9 \\
\hline & 40 & $\begin{array}{l}7-5 \\
7-6 \\
7-7\end{array}$ & $\begin{array}{c}\mathrm{RT} \\
1000 \mathrm{~F} \\
1000 \mathrm{~F} / \\
200 \mathrm{ks} \mathrm{i}\end{array}$ & $\begin{array}{l}254.7 \\
214.7\end{array}$ & $\begin{array}{l}253.5 \\
210.1\end{array}$ & $\begin{array}{r}7.5 \\
7.5 \\
10.0\end{array}$ & \multicolumn{2}{|l|}{$\begin{array}{r}32.1 \\
24.6 \\
8.8\end{array}$} & 47.5 \\
\hline & 50 & $\begin{array}{l}8-5 \\
8-6 \\
8-7\end{array}$ & $\begin{array}{c}\mathrm{RT} \\
1000 \mathrm{~F} \\
1000 \mathrm{~F} / \\
209 \mathrm{ksi}\end{array}$ & $\begin{array}{l}269.3 \\
227.9\end{array}$ & $\begin{array}{l}260.5 \\
279.6\end{array}$ & $\begin{array}{r}5.5 \\
5.0 \\
11.4\end{array}$ & \multicolumn{2}{|l|}{$\begin{array}{l}19.6 \\
17.2 \\
20.7\end{array}$} & 49.7 \\
\hline
\end{tabular}


Table 7

Mechanical Properties after $1275 \mathrm{~F} / 8 \mathrm{nr} / \mathrm{FC} 100 \mathrm{~F} / \mathrm{hr}$ to $1150 \mathrm{~F} / 8 \mathrm{hr} / \mathrm{AC}$ $.375^{\text {" Round }}$

\begin{tabular}{|c|c|c|c|c|c|c|c|c|c|}
\hline $\begin{array}{c}\text { SOLUTION } \\
\text { TEMP. } \\
\text { F }\end{array}$ & $\begin{array}{c}\text { NOMINAL } \\
\text { COLD } \\
\text { REDUCTION } \\
\% \\
\end{array}$ & $\begin{array}{l}\text { SPECIMEN } \\
\text { IDENTITY } \\
\end{array}$ & $\begin{array}{c}\text { TEST } \\
\text { CONDITIONS } \\
\end{array}$ & $\begin{array}{l}\text { UTS } \\
\text { ksi } \\
\end{array}$ & $\begin{array}{l}.2 Y S \\
\mathrm{ksi} \\
\end{array}$ & $\begin{array}{c}\text { ELONG. } \\
\%\end{array}$ & $\begin{array}{l}\text { R.A. } \\
\% \\
\end{array}$ & $\begin{array}{c}\text { STRESS } \\
\text { RUPTURE } \\
\text { LIFE } \\
\text { hr } \\
\end{array}$ & $\underline{\mathrm{R}}$ \\
\hline \multirow[t]{4}{*}{1750} & 20 & $\begin{array}{l}A-5 \\
A-6 \\
A-7\end{array}$ & $\begin{array}{r}\mathrm{RT} \\
1000 \mathrm{~F} \\
1000 \mathrm{~F} / \\
183 \mathrm{ks}\end{array}$ & $\begin{array}{l}235.7 \\
206.8\end{array}$ & $\begin{array}{l}220.4 \\
192.4\end{array}$ & $\begin{array}{r}8.5 \\
9.5 \\
10.0\end{array}$ & $\begin{array}{l}27.3 \\
31.9 \\
24.1\end{array}$ & 68.9 & 46.5 \\
\hline & 30 & $\begin{array}{l}B-5 \\
B-6 \\
B-7\end{array}$ & $\begin{array}{c}\mathrm{RT} \\
1000 \mathrm{~F} \\
1000 \mathrm{~F} / \\
200 \mathrm{ks}\end{array}$ & $\begin{array}{l}250.5 \\
219.2\end{array}$ & $\begin{array}{l}240.5 \\
210.4\end{array}$ & $\begin{array}{r}8.5 \\
7.0 \\
12.9\end{array}$ & $\begin{array}{l}27.9 \\
25.3 \\
28.1\end{array}$ & 60.0 & 47.6 \\
\hline & 40 & $\begin{array}{l}C-5 \\
C-6 \\
C-7\end{array}$ & $\begin{array}{c}\mathrm{RT} \\
1000 \mathrm{~F} \\
1000 \mathrm{~F} / \\
214 \mathrm{ksi}\end{array}$ & $\begin{array}{l}276.8 \\
238.6\end{array}$ & $\begin{array}{l}268.0 \\
224.9\end{array}$ & $\begin{array}{r}5.7 \\
5.7 \\
12.9\end{array}$ & $\begin{array}{l}19.6 \\
23.3 \\
25.3\end{array}$ & 14.1 & 48.2 \\
\hline & 50 & $\begin{array}{l}D-5 \\
D-6 \\
D-7\end{array}$ & $\begin{array}{r}\mathrm{RT} \\
1000 \mathrm{~F} \\
1000 \mathrm{~F} / \\
224 \mathrm{ksi}\end{array}$ & $\begin{array}{l}287.4 \\
243.2\end{array}$ & $\begin{array}{l}285.4 \\
236.0\end{array}$ & $\begin{array}{r}7.1 \\
4.3 \\
11.4\end{array}$ & $\begin{array}{l}13.4 \\
19.6 \\
17.4\end{array}$ & 1.4 & 50.3 \\
\hline \multirow[t]{4}{*}{1840} & 20 & $\begin{array}{l}E-5 \\
E-6 \\
E-7\end{array}$ & $\begin{array}{r}\mathrm{RT} \\
1000 \mathrm{~F} \\
1000 \mathrm{~F} / \\
162 \mathrm{ks}\end{array}$ & $\begin{array}{l}212.4 \\
183.3\end{array}$ & $\begin{array}{l}198.4 \\
171.0\end{array}$ & $\begin{array}{l}15.0 \\
14.5\end{array}$ & $\begin{array}{c}41.9 \\
41.7 \\
\text { (In tes }\end{array}$ & & 44.7 \\
\hline & 30 & $\begin{array}{l}F-5 \\
F-6 \\
F-7\end{array}$ & $\begin{array}{r}\mathrm{RT} \\
1000 \mathrm{~F} \\
1000 \mathrm{~F} / \\
184 \mathrm{ksi}\end{array}$ & $\begin{array}{l}235.5 \\
202.4\end{array}$ & $\begin{array}{l}228.5 \\
193.9\end{array}$ & $\begin{array}{l}11.0 \\
10.0 \\
17.0\end{array}$ & $\begin{array}{r}39.5 \\
33.9 \\
7.6\end{array}$ & 624.0 & 47.3 \\
\hline & 40 & $\begin{array}{l}G-5 \\
G-6 \\
G-7\end{array}$ & $\begin{array}{r}\text { RT } \\
1000 \mathrm{~F} \\
1000 \mathrm{~F} / \\
206 \mathrm{ksi}\end{array}$ & $\begin{array}{l}257.8 \\
216.9\end{array}$ & $\begin{array}{l}253.0 \\
216.9\end{array}$ & $\begin{array}{l}7.1 \\
5.7 \\
7.1\end{array}$ & $\begin{array}{l}30.1 \\
27.3 \\
16.1\end{array}$ & 14.7 & 48.3 \\
\hline & 50 & $\begin{array}{l}H-5 \\
H-6 \\
H-7\end{array}$ & $\begin{array}{c}\mathrm{RT} \\
1000 \mathrm{~F} \\
1000 \mathrm{~F} / \\
216 \mathrm{ks} i\end{array}$ & $\begin{array}{l}271.5 \\
232.9\end{array}$ & $\begin{array}{l}267.1 \\
226.9\end{array}$ & $\begin{array}{r}7.1 \\
7.1 \\
10.0\end{array}$ & $\begin{array}{l}19.3 \\
23.3 \\
18.3\end{array}$ & 25.6 & 48.7 \\
\hline
\end{tabular}


Table 8

$\frac{\text { Mechanical Properties after } 1450 \mathrm{~F} / 4 \mathrm{hr} / \mathrm{WQ}+1150 \mathrm{~F} / 8 \mathrm{hr} / \mathrm{AC}}{.875^{\prime \prime} \text { Round }}$

\begin{tabular}{|c|c|c|c|c|c|c|c|c|c|}
\hline $\begin{array}{c}\text { SOLUTION } \\
\text { TEMP. } \\
\mathrm{F}\end{array}$ & $\begin{array}{c}\text { NOMINAL } \\
\text { COLD } \\
\text { REDUCTION } \\
\% \\
\end{array}$ & $\begin{array}{l}\text { SPECIMEN } \\
\text { IDENTITY }\end{array}$ & $\begin{array}{c}\text { TEST } \\
\text { CONDITIONS } \\
\end{array}$ & $\begin{array}{l}\text { UTS } \\
\text { ksi } \\
\end{array}$ & $\begin{array}{l}.2 Y S \\
\mathrm{ksi} \\
\end{array}$ & $\begin{array}{c}\text { ELONG. } \\
\% \\
\end{array}$ & $\begin{array}{l}\text { R.A. } \\
\%\end{array}$ & $\begin{array}{c}\text { STRESS } \\
\text { UUPTURE } \\
\text { LIFE } \\
\text { hr } \\
\end{array}$ & $\mathrm{R}_{\mathrm{c}}$ \\
\hline \multirow[t]{4}{*}{1750} & 20 & $\begin{array}{l}7-8 \\
7-9 \\
1-10\end{array}$ & $\begin{array}{c}\mathrm{RT} \\
1000 \mathrm{~F} \\
1000 \mathrm{~F} / \\
155 \mathrm{ks}\end{array}$ & $\begin{array}{l}218.5 \\
194.0\end{array}$ & $\begin{array}{l}196.4 \\
162.3\end{array}$ & $\begin{array}{l}18.5 \\
16.0\end{array}$ & \multicolumn{2}{|c|}{$\begin{array}{l}35.1 \\
38.3 \\
\text { (In test) }\end{array}$} & 44.0 \\
\hline & 30 & $\begin{array}{l}2-8 \\
2-9 \\
2-10\end{array}$ & $\begin{array}{c}\mathrm{RT} \\
1000 \mathrm{~F} \\
1000 \mathrm{~F} / \\
174 \mathrm{ksi}\end{array}$ & $\begin{array}{l}228.1 \\
204.8\end{array}$ & $\begin{array}{l}210.4 \\
182.9\end{array}$ & $\begin{array}{l}14.5 \\
13.0\end{array}$ & \multicolumn{2}{|c|}{$\begin{array}{l}30.7 \\
33.8 \\
\text { (In test) }\end{array}$} & 45.5 \\
\hline & 40 & $\begin{array}{l}3-8 \\
3-9 \\
3-10\end{array}$ & $\begin{array}{c}\mathrm{RT} \\
1000 \mathrm{~F} \\
1000 \mathrm{~F} / \\
180 \mathrm{ks} 1\end{array}$ & $\begin{array}{l}236.1 \\
205.6\end{array}$ & $\begin{array}{l}218.4 \\
188.9\end{array}$ & $\begin{array}{l}10.0 \\
10.0 \\
10.0\end{array}$ & \multicolumn{2}{|l|}{$\begin{array}{l}23.0 \\
25.8 \\
15.7\end{array}$} & 47.5 \\
\hline & 50 & $\begin{array}{l}4-8 \\
4-9 \\
4-10\end{array}$ & $\begin{array}{c}\text { RT } \\
1000 \mathrm{~F} \\
1000 \mathrm{~F} / \\
183 \mathrm{ksi}\end{array}$ & $\begin{array}{l}244.9 \\
218.8\end{array}$ & $\begin{array}{l}220.7 \\
192.7\end{array}$ & $\begin{array}{r}7.5 \\
9.0 \\
22.9\end{array}$ & \multicolumn{2}{|l|}{$\begin{array}{l}18.9 \\
25.5 \\
31.7\end{array}$} & 47.5 \\
\hline 1840 & 20 & $\begin{array}{l}5-8 \\
5-9 \\
5-10\end{array}$ & $\begin{array}{c}\text { RT } \\
1000 \mathrm{~F} \\
1000 \mathrm{~F} / \\
114 \mathrm{ksi}\end{array}$ & $\begin{array}{l}217.6 \\
181.2\end{array}$ & $\begin{array}{l}180.9 \\
151.3\end{array}$ & $\begin{array}{l}20.0 \\
17.0\end{array}$ & \multicolumn{2}{|c|}{$\begin{array}{l}37.0 \\
39.5 \\
\text { (In test) }\end{array}$} & 44.5 \\
\hline & 30 & $\begin{array}{l}6-8 \\
6-9 \\
6-10\end{array}$ & $\begin{array}{c}\mathrm{RT} \\
1000 \mathrm{~F} \\
1000 \mathrm{~F} / \\
164 \mathrm{ksi}\end{array}$ & $\begin{array}{l}221.3 \\
198.0\end{array}$ & $\begin{array}{l}205.2 \\
172.3\end{array}$ & $\begin{array}{l}14.0 \\
13.0\end{array}$ & \multicolumn{2}{|c|}{$\begin{array}{l}30.3 \\
30.7 \\
\text { (In test) }\end{array}$} & 45.0 \\
\hline & 40 & $\begin{array}{l}7-8 \\
7-9 \\
7-10\end{array}$ & $\begin{array}{r}R T \\
1000 \mathrm{~F} \\
1000 \mathrm{~F} / \\
171 \mathrm{ksi}\end{array}$ & $\begin{array}{l}230.7 \\
202.8\end{array}$ & $\begin{array}{l}213.6 \\
179.6\end{array}$ & $\begin{array}{r}11.0 \\
12.0 \\
8.6\end{array}$ & \multicolumn{2}{|l|}{$\begin{array}{l}23.1 \\
28.9 \\
10.9\end{array}$} & 46.3 \\
\hline & 50 & $\begin{array}{l}8-8 \\
8-9 \\
8-10\end{array}$ & $\begin{array}{c}\mathrm{RT} \\
1000 \mathrm{~F} \\
1000 \mathrm{~F} / \\
189 \mathrm{ksi}\end{array}$ & $\begin{array}{l}254.9 \\
219.6\end{array}$ & $\begin{array}{l}244.5 \\
198.4\end{array}$ & $\begin{array}{r}8.5 \\
9.0 \\
20.0\end{array}$ & \multicolumn{2}{|l|}{$\begin{array}{l}20.2 \\
26.7 \\
27.6\end{array}$} & 47.5 \\
\hline
\end{tabular}


Table 9

Mechanical Properties after 1450F/4 hr/WQ $+1150 \mathrm{~F} / 8 \mathrm{hr} / \mathrm{AC}$ $.375^{\prime \prime}$ Round

\begin{tabular}{|c|c|c|c|c|c|c|c|c|c|}
\hline $\begin{array}{l}\text { SOLUTION } \\
\text { TEMP. } \\
\mathrm{F} \\
\end{array}$ & $\begin{array}{c}\text { NOMINAL } \\
\text { COLD } \\
\text { REDUCTION } \\
\% \\
\end{array}$ & $\begin{array}{l}\text { SPECIMEN } \\
\text { IDENTITY } \\
\end{array}$ & $\begin{array}{c}\text { TEST } \\
\text { CONDITIONS } \\
\end{array}$ & $\begin{array}{l}\text { UTS } \\
\text { ksi } \\
\end{array}$ & $\begin{array}{r}.2 Y S \\
k s i \\
\end{array}$ & $\begin{array}{c}\text { ELONG. } \\
\%\end{array}$ & $\begin{array}{r}\text { R.A. } \\
\% \\
\end{array}$ & $\begin{array}{c}\text { STRESS } \\
\text { RUPTURE } \\
\text { LIFE } \\
h r \\
\end{array}$ & $\underline{\mathrm{R}_{\mathrm{C}}}$ \\
\hline \multirow[t]{4}{*}{1750} & 20 & $\begin{array}{l}A-8 \\
A-9 \\
A-10\end{array}$ & $\begin{array}{c}\mathrm{RT} \\
1000 \mathrm{~F} \\
1000 \mathrm{~F} / \\
167 \mathrm{ksi}\end{array}$ & $\begin{array}{l}230.0 \\
204.1\end{array}$ & $\begin{array}{l}199.2 \\
175.5\end{array}$ & $\begin{array}{l}14.3 \\
10.0 \\
21.4\end{array}$ & $\begin{array}{l}27.2 \\
18.6 \\
35.3\end{array}$ & 207.5 & 44.0 \\
\hline & 30 & $\begin{array}{l}\text { B-8 } \\
\text { B-9 } \\
B-10\end{array}$ & $\begin{array}{r}\text { RT } \\
1000 \mathrm{~F} \\
1000 \mathrm{~F} / \\
174 \mathrm{ks}\end{array}$ & $\begin{array}{l}235.3 \\
212.0\end{array}$ & $\begin{array}{l}216.9 \\
182.7\end{array}$ & $\begin{array}{r}15.7 \\
9.3 \\
14.3\end{array}$ & $\begin{array}{l}30.9 \\
25.3 \\
32.7\end{array}$ & 93.3 & 44.6 \\
\hline & 40 & $\begin{array}{l}C-8 \\
C-9 \\
C-10\end{array}$ & $\begin{array}{c}\text { RT } \\
1000 \mathrm{~F} \\
1000 \mathrm{~F} / \\
178 \mathrm{ksi}\end{array}$ & $\begin{array}{l}239.4 \\
209.6\end{array}$ & $\begin{array}{l}216.9 \\
186.7\end{array}$ & $\begin{array}{l}12.1 \\
11.4 \\
35.7\end{array}$ & $\begin{array}{l}14.7 \\
19.3 \\
21.0\end{array}$ & $4.5^{*}$ & 44.8 \\
\hline & 50 & $\begin{array}{l}D-8 \\
D-9 \\
D-10\end{array}$ & $\begin{array}{r}\mathrm{RT} \\
1000 \mathrm{~F} \\
1000 \mathrm{~F} / \\
184 \mathrm{ks} \mathrm{i}\end{array}$ & $\begin{array}{l}247.4 \\
217.7\end{array}$ & $\begin{array}{l}228.9 \\
192.8\end{array}$ & $\begin{array}{r}8.6 \\
8.6 \\
21.4\end{array}$ & $\begin{array}{l}17.3 \\
20.0 \\
26.1\end{array}$ & $18.8^{*}$ & 46.0 \\
\hline \multirow[t]{4}{*}{1840} & 20 & $\begin{array}{l}E-8 \\
E-9 \\
E-10\end{array}$ & $\begin{array}{r}\mathrm{RT} \\
1000 \mathrm{~F} \\
1000 \mathrm{~F} / \\
146 \mathrm{ksi}\end{array}$ & $\begin{array}{l}213.7 \\
185.7\end{array}$ & $\begin{array}{l}181.5 \\
153.5\end{array}$ & $\begin{array}{l}25.7 \\
14.3\end{array}$ & $\begin{array}{c}37.9 \\
36.7 \\
\text { (In test) }\end{array}$ & & 44.6 \\
\hline & 30 & $\begin{array}{l}F-8 \\
F-9 \\
F-70\end{array}$ & $\begin{array}{c}\mathrm{RT} \\
1000 \mathrm{~F} \\
1000 \mathrm{~F} / \\
169 \mathrm{ksi}\end{array}$ & $\begin{array}{l}224.9 \\
195.2\end{array}$ & $\begin{array}{l}202.8 \\
177.7\end{array}$ & $\begin{array}{l}15.7 \\
14.3\end{array}$ & $\begin{array}{c}30.9 \\
34.5 \\
\text { (In test) }\end{array}$ & & 44.8 \\
\hline & 40 & $\begin{array}{l}G-8 \\
G-9 \\
G-10\end{array}$ & $\begin{array}{l}\mathrm{RT} \\
1000 \mathrm{~F} \\
1000 \mathrm{~F} / \\
169 \mathrm{ksi}\end{array}$ & $\begin{array}{l}233.9 \\
205.0\end{array}$ & $\begin{array}{l}213.7 \\
177.4\end{array}$ & $\begin{array}{l}12.9 \\
11.4\end{array}$ & $\begin{array}{c}23.0 \\
25.0 \\
\text { (In test) }\end{array}$ & & 46.0 \\
\hline & 50 & $\begin{array}{l}H-8 \\
H-9 \\
H-10\end{array}$ & $\begin{array}{l}\mathrm{RT} \\
1000 \mathrm{~F} \\
1000 \mathrm{~F} / \\
182 \mathrm{ksi}\end{array}$ & $\begin{array}{l}239.9 \\
275.6\end{array}$ & $\begin{array}{l}217.7 \\
191.4\end{array}$ & $\begin{array}{r}9.3 \\
11.4 \\
17.1\end{array}$ & $\begin{array}{l}14.9 \\
22.6 \\
28.9\end{array}$ & 50.4 & 46.2 \\
\hline
\end{tabular}

* Temperature overrode $100^{\circ} \mathrm{F}$. 
solutioning at $1750 \mathrm{~F}$ results in higher tensile strengths than does the 1840F solution, the difference is not large and is insignificant at 20 to 30 percent cold work. The $1840 \mathrm{~F}$ solution treatment results in slightly higher tensile elongations at the 40 and 50 percent reduction levels.

In general, the $1750 \mathrm{~F}$ pre-draw solution treatment a1so produces higher yield strengths and rupture elongations and lower rupture strengths than does the 1840F treatment. These observations hold true for both bar sizes and all aging treatments. These improvements are related to the finer grain size that results from the 1750F solution trealment.

The above data indicate that the selection of solution treating temperature (1750F or 1840F) is not too critical. If tensile strength is the overriding property then the lower temperature will produce better results; however, if tensile ductility is of prime concern, the $1840 \mathrm{~F}$ treatment would be justified. In addition to producing improved tensile ductilities, use of the 1840F solution treatment will tend to improve drawability.

\section{Effect of Size}

In almost a11 instances, specimens from the $.375^{\prime \prime}$ round bars exhibit higher strengths than specimens from the $.875^{\prime \prime}$ round bars, Figure 14. This strength differential diminishes as the amount of reduction increases. This behavior is common in cold worked materials because as bar size decreases (or reduction increases) the penetration of cold work becomes deeper and more uniform.

Bar size had no significant influence on tensile ductility, Figure 14 . However, the .875" bars exhibit higher rupture strengths and lower rupture ductilities than the $.375^{\prime \prime}$ bars. The latter effects are attributed in part to the slightly coarser grain size of the larger bars.

\section{Microstructures}

Light and electron micrographs showing structures typical of the bars in the solution treated and cold reduced condition are shown in Figure 15. Note that the specimens solution treated at $1750 \mathrm{~F}$ contain $\mathrm{Ni}_{3} \mathrm{Cb}$ at the grain boundaries; whereas, those solutioned at $1840 \mathrm{~F}$ do not. Strain lines were clearly evident on specimens reduced more than 30 percent.

Structures typical of specimens given the three aging treatments are shown in Figures 16,17 and 18 .

Examination of these figures leads to the following observations:

(1) As the amount of cold work increases, the rate of precipitation increases regardless of the aging cycle used.

(2) For a given degree of cold reduction, the size and amount of platelet particles increases as the initial aging temperature increases from $1275 \mathrm{~F}$ to $1325 \mathrm{~F}$ to $1450 \mathrm{~F}$. It also appears that preferential platelet precipitation occurs in the strain 1 ines. Although no X-ray or electron diffraction work was carried out, it is suspected from prior work on 718 (3) that the platelet phase is $\mathrm{Ni}_{3} \mathrm{Cb}$. The abunciant precipitation of this phase would compete with $r^{\prime}$ precipitation and thereby lower the amount of columbium available for strengthening purposes. This would explain the "overaging" effect seen in the yield strength, Figure 9. 
(3) Although it is not evident in the photomicrographs, it is speculated that the size of the r'particles increases and their volume decreases with increasing aging temperature. This would lead to lower tensile strengths and higher tensile elongations because of a less than optimum size/volume ratio. The leveling off of the yield strength at 50 percent reduction after the $1325 \mathrm{~F}+1150 \mathrm{~F}$ and $1450 \mathrm{~F}+1150 \mathrm{~F}$ ages suggests that these specimens are beginning to overage.

(4) Specimens solutioned at 1840F exhibit a coarser grain size than those solutioned at 1750F. This in part accounts for the lower tensile strengths and higher tensile ductilities of the specimens solutioned at 1840F. The light photographs show that the specimens solutioned at $1750 \mathrm{~F}$ age more readily than the specimens solutioned at 1840F. This is due to the fact that the $1750 \mathrm{~F}$ treatment does not completely solution the material.

CONCLUSIONS

The following conclusions are based on the results obtained in this study:

(1) Regardless of solution treating temperature, bar size or aging treatment, increased cold reduction results in (a) higher tensile and yield strengths, (b) higher rupture ductilities, (c) lower tensile ductilities and (d) lower rupture strengths. These effects are due to residual stress and stress induced precipitation.

(2) Mechanical properties vary significantly with aging cycle. For a given reduction, the size and amount of platelet particles increase as the initial aging temperature increases from $1275 \mathrm{~F}$ to $1325 \mathrm{~F}$ to $1450 \mathrm{~F}$. At the 50 percent cold reduction level the specimens aged at $1325 \mathrm{~F}+1150 \mathrm{~F}$ and $1450 \mathrm{~F}+1150 \mathrm{~F}$ show evidence of overaging. The $1275 \mathrm{~F}+1150 \mathrm{~F}$ cycle produces the highest tensile and yield strengths; whereas, the $1450 \mathrm{~F}+1150 \mathrm{~F}$ cycle results in the highest tensile ductilities. However, the best combination of these properties, tensile strength and ductility, is obtained through use of the conventional $1325 \mathrm{~F}+1150 \mathrm{~F}$ cycle. Although the stress rupture data are incomplete, it appears that the $1450 \mathrm{~F}+1150 \mathrm{~F}$ cycle produces the best balance of rupture strength and rupture elongation. However, considering al1 mechanical properties, the best balance of properties is obtained by use of the $1325 \mathrm{~F}+1150 \mathrm{~F}$ age.

(3) Pre-draw solution heat treatment does not have a significant effect on properties. The $1750 \mathrm{~F}$ treatment results in higher tensile strengths and rupture elongations, but lower tensile ductilities and rupture strengths than the $1840 \mathrm{~F}$ treatment. These effects are related to the finer grain size that results from the 1750F solution treatment.

(4) The smaller bars (.375" RD.) exhibit higher tensile and yield strengths than the larger (.875" RD.) bars, but this effect diminishes as percent reduction increases. Bar size had no effect on tensile ductility. The $.875^{\prime \prime}$ round bars exhibit higher rupture strengths and lower rupture ductilities than the $.375^{\prime \prime}$ round bars. The above effects are attributed to the greater penetration of cold work on smaller diameter bars and to the finer grain size exhibited by the smaller bars. 


\section{REFERENCES}

1. H. L. Eiselstein, "Age-Hardenable Nickel Alloy", U.S. Patent 3,046,108, Ju7y 24, 1962.

2. R. A. Heacox, Unpublished Research. Latrobe Steel Company, 1972.

3. J. P. Stroup and R. A. Heacox, "Effect of Grain Size Variation on the Long Time Stability of Alloy 718", First International Symposium on Structural Stability on Superalloys, Seven Springs, Pa., September 1968. 


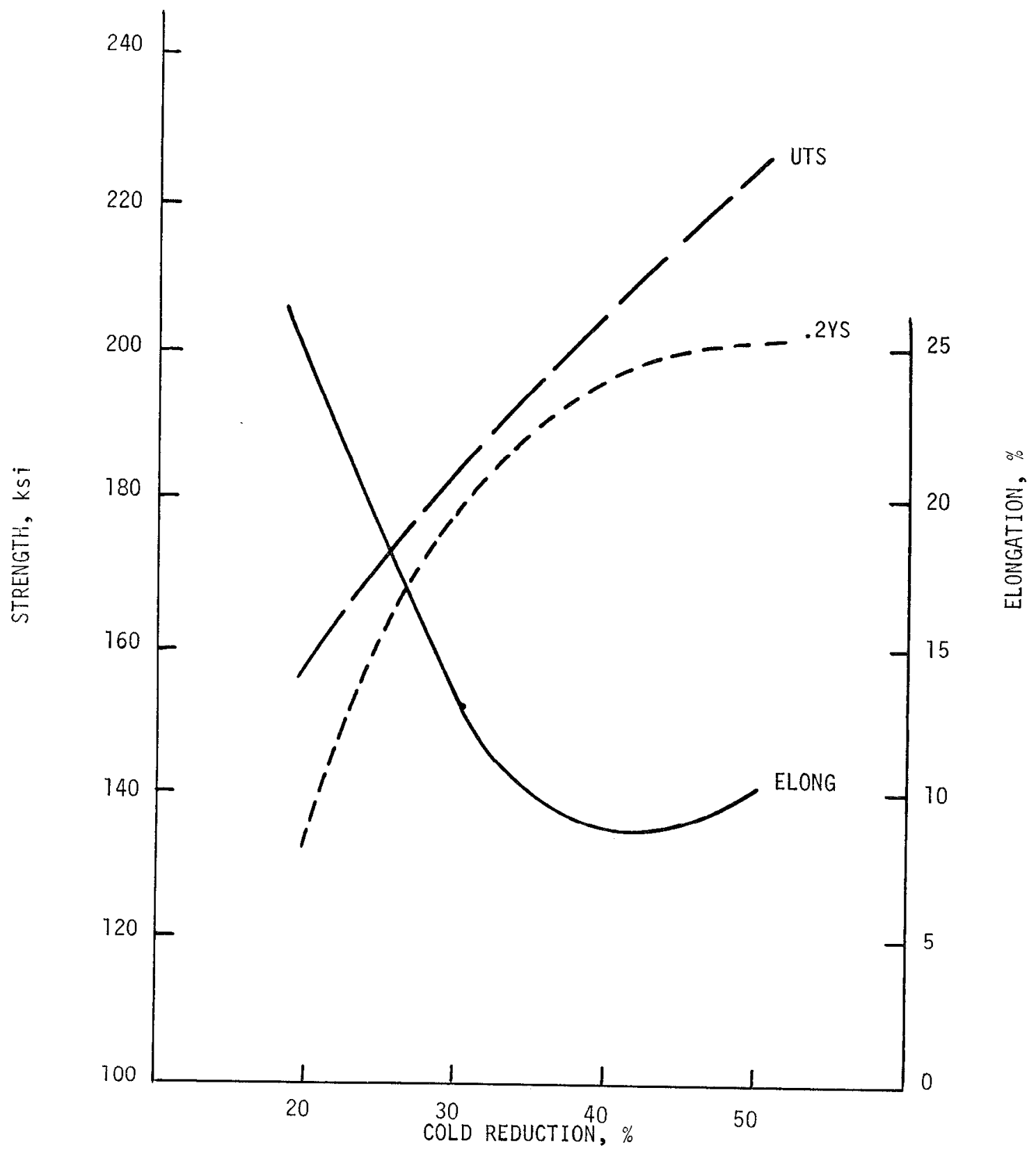

FIGURE 1. EFFECT OF COLD REDUCTION ON ROOM - TEMPERATURE TENSILE PROPERTIES OF .875" ROUND BARS IN COLD REDUCED CONDITION. 1750F PRIOR SOLUTION TREATMENT. 


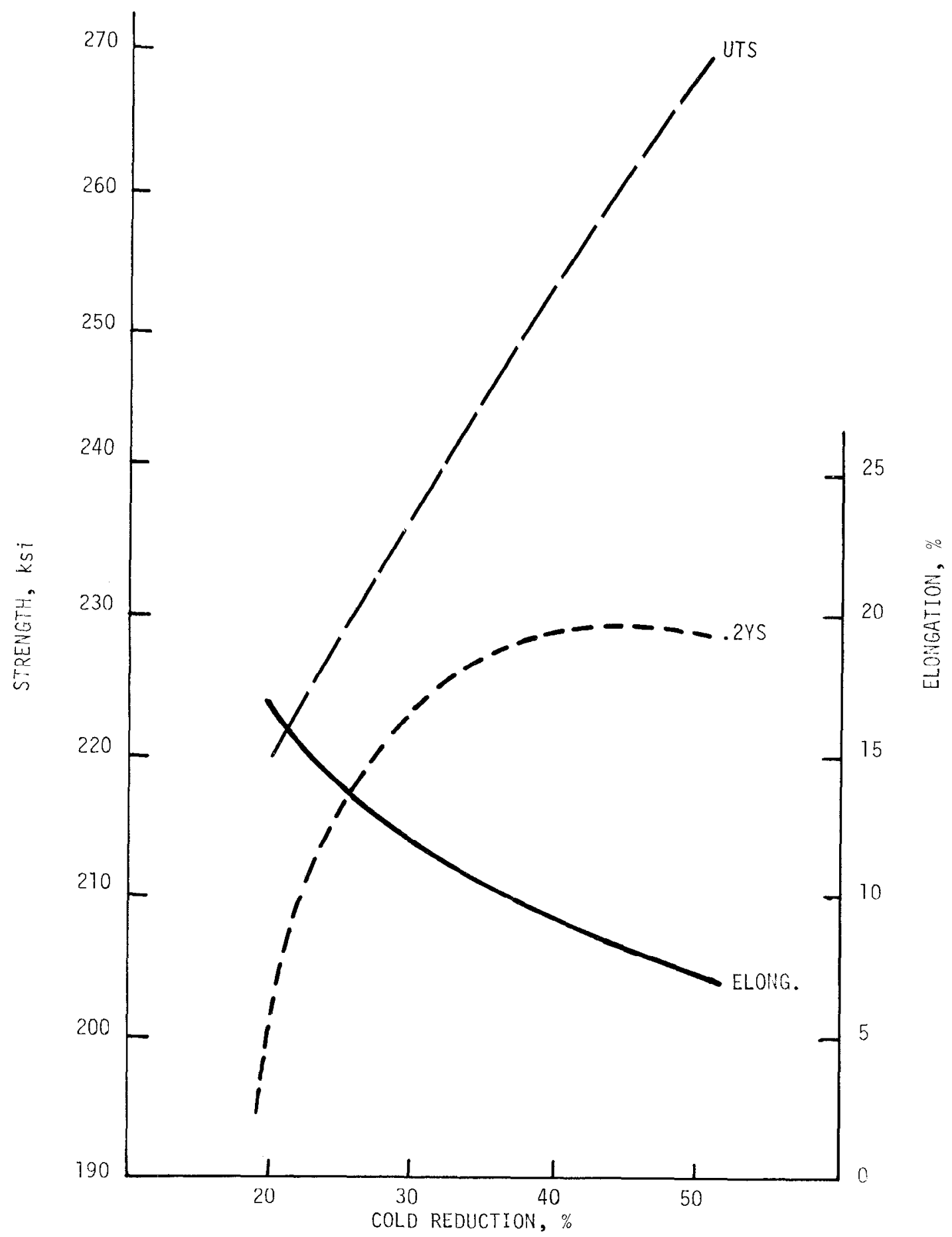

FIGURE 2. EFFECT OF COLD REDUCTION ON ROOM TEMPERATURE TENSILE PRODERTIE: OF .875" ROUND BARS AFTER 1325F+1150F AGE. 1750F PRIOR SOIUTION TREATMENT. 


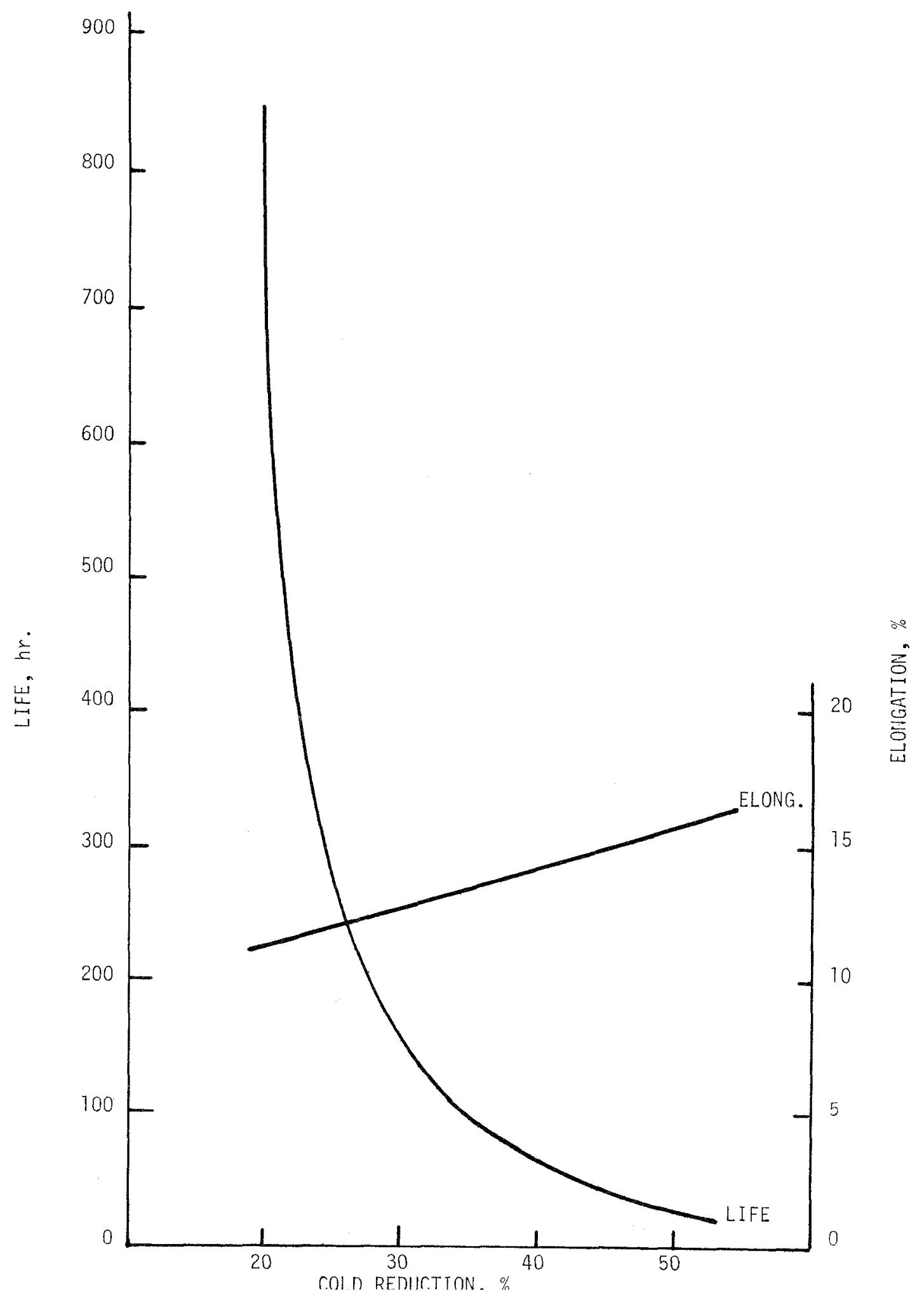

FIGURE 3. EFFECT OF COLD REDUCTION OII 1000F STRESS RUPTURE PROPERTIES OF $.875^{\prime \prime}$ ROUND BARS AFTER 1325F+7150F AGE. TEST STRESS EQUALS $95 \%$ OF $7000 F$ YIELD STRENGTH. T750F PRIOR SOLUTION TREATMENT. 


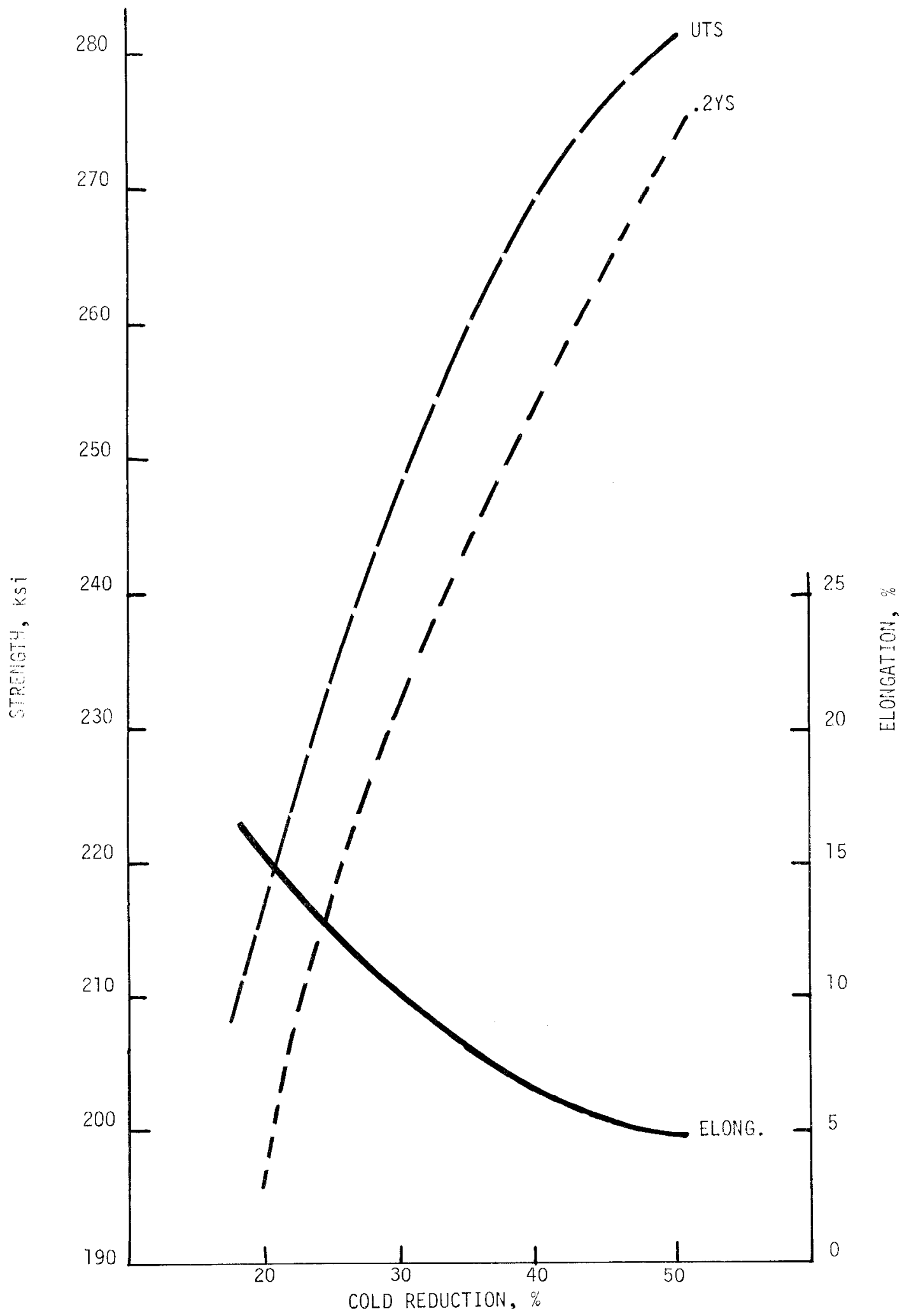

FIGURE 4. EFFECT OF COLD REDUCTION ON ROOM TEMPERATURE TENSILE PROFERTIES OF $.875 "$ ROUND BARS AFTER $1275 \mathrm{~F}+1750 \mathrm{~F}$ AGE. 1750F PRIOR SOLUTION TREATMENT. 


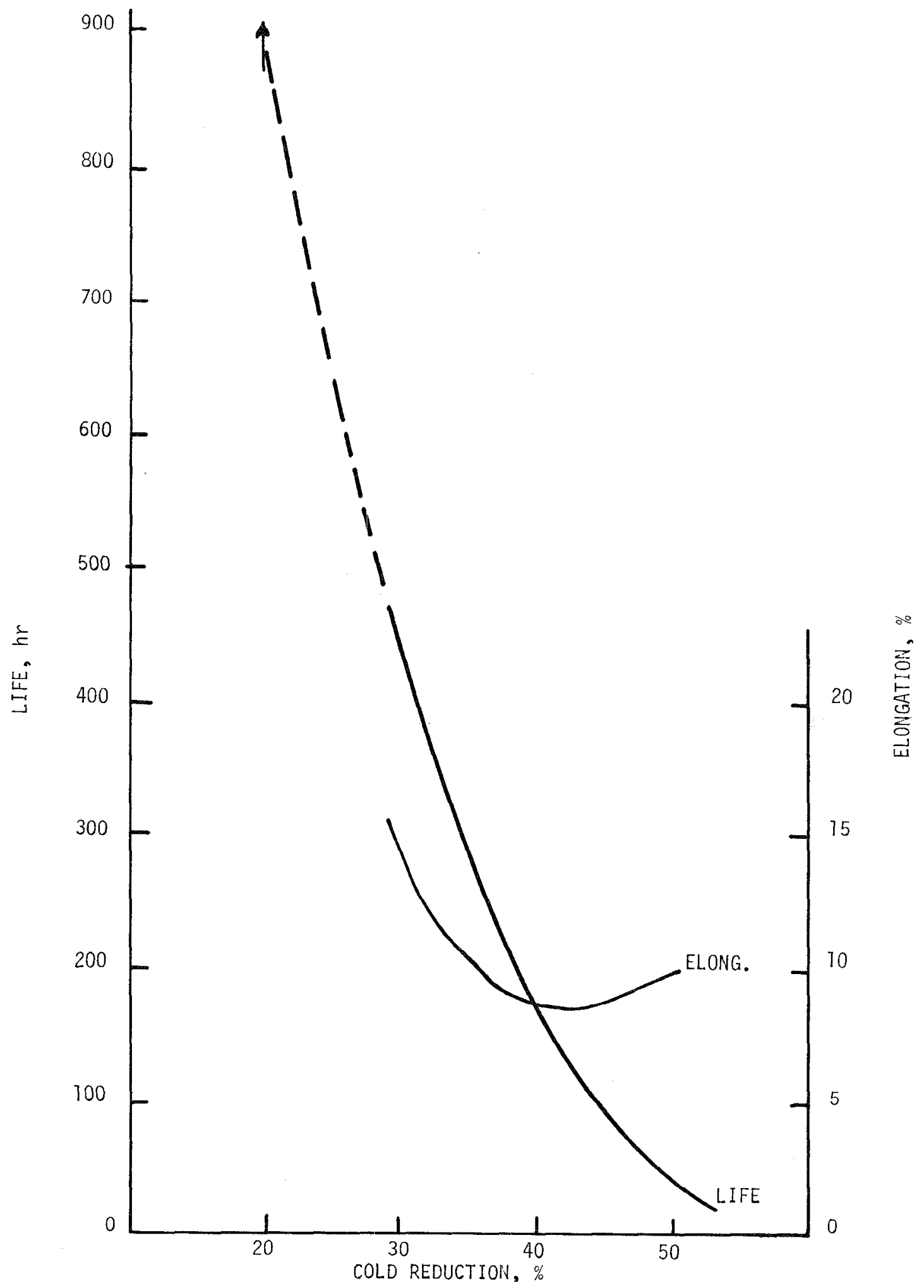

FIGURE 5. EFFECT OF COLD REDUCTION ON 1000F STRESS RUPTURE PROPERTIES OF .875" ROUND BARS AFTER 1275F+1750F AGE. TEST STRESS EQUALS 95\% OF 1000F YIELD STRENGTH. 1750F PRIOR SOLUTION TREATMENT. 


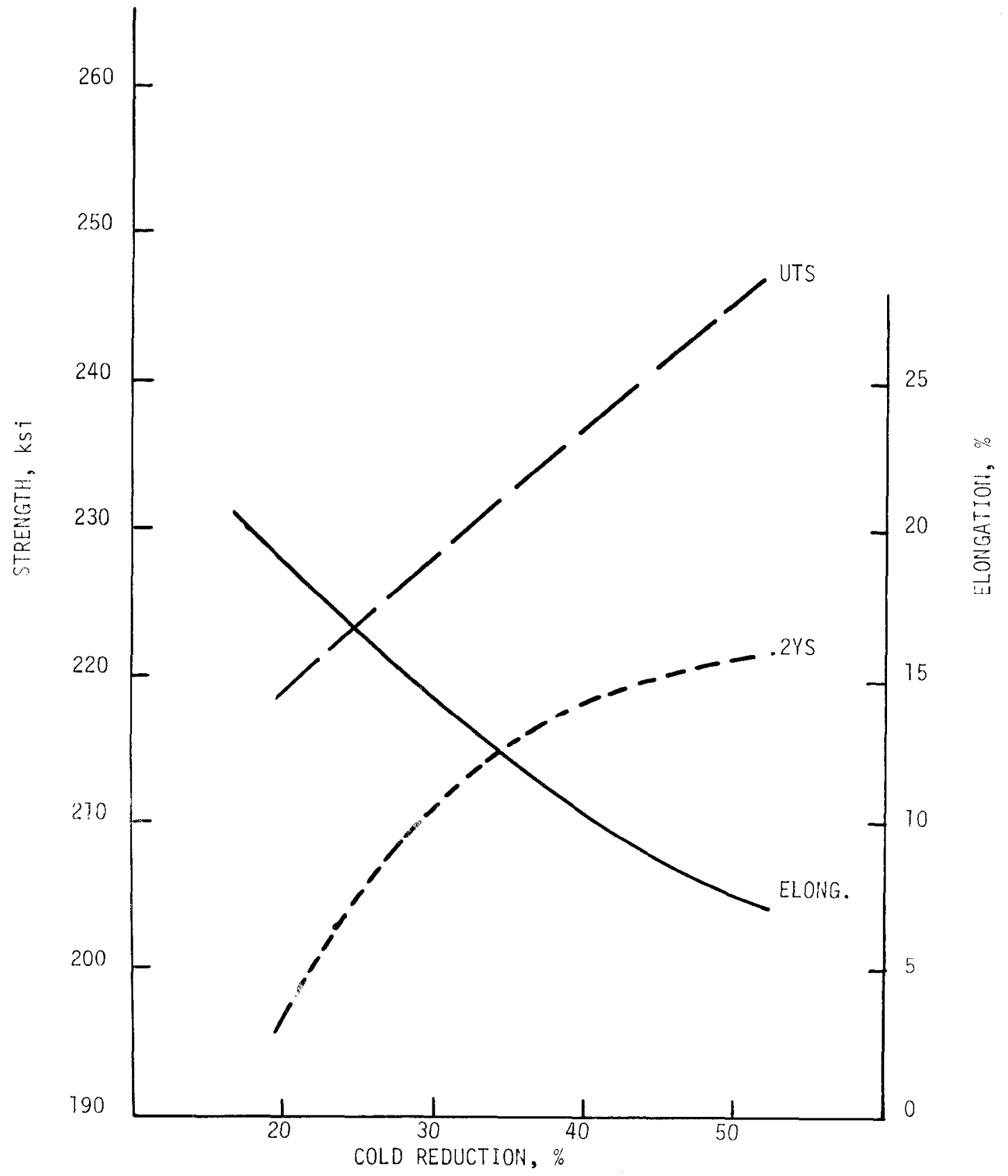

FIGURE 6. EFFECT OF COLD REDUCTION ON ROOM TEMPERATURE TENSILE PROPERTIES OF .875" ROUND BARS AFTER 1450F+ 1150F AGE. 1750F PRIOR SOLUT'OH TREATMENT. 


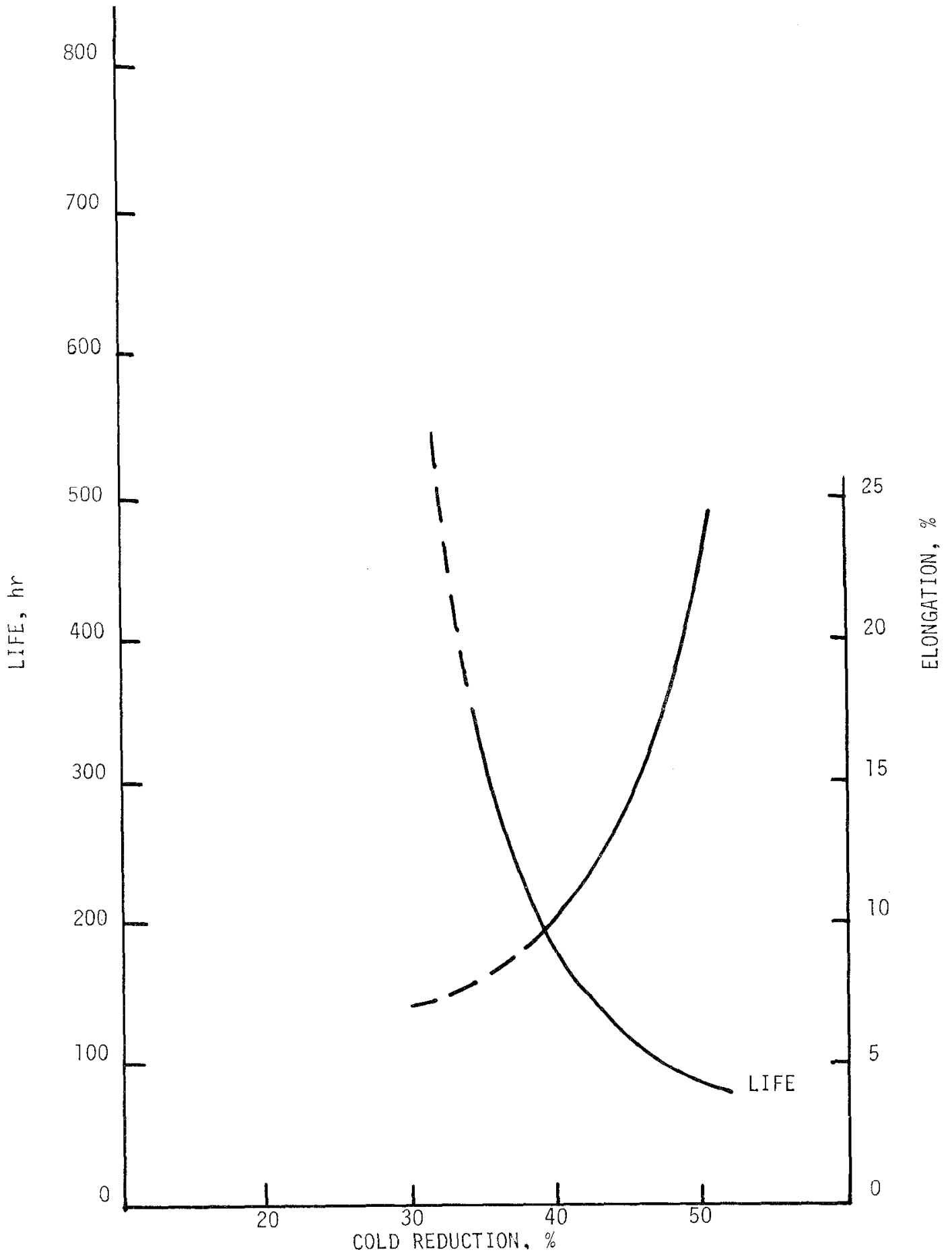

FIGURE 7. EFFECT OF COLD REDUCTION ON 1000F STRESS RUPTURE PROPERTIES OF $.875^{11}$ ROUND BARS AFTER 1450F+1150F AGE. TEST STRENGTH EOUALS $95 \%$ of $1000 \mathrm{~F} \vee$ IELD STRENGTH. 1750F PRIOR SOLUTION TREATMENT. 

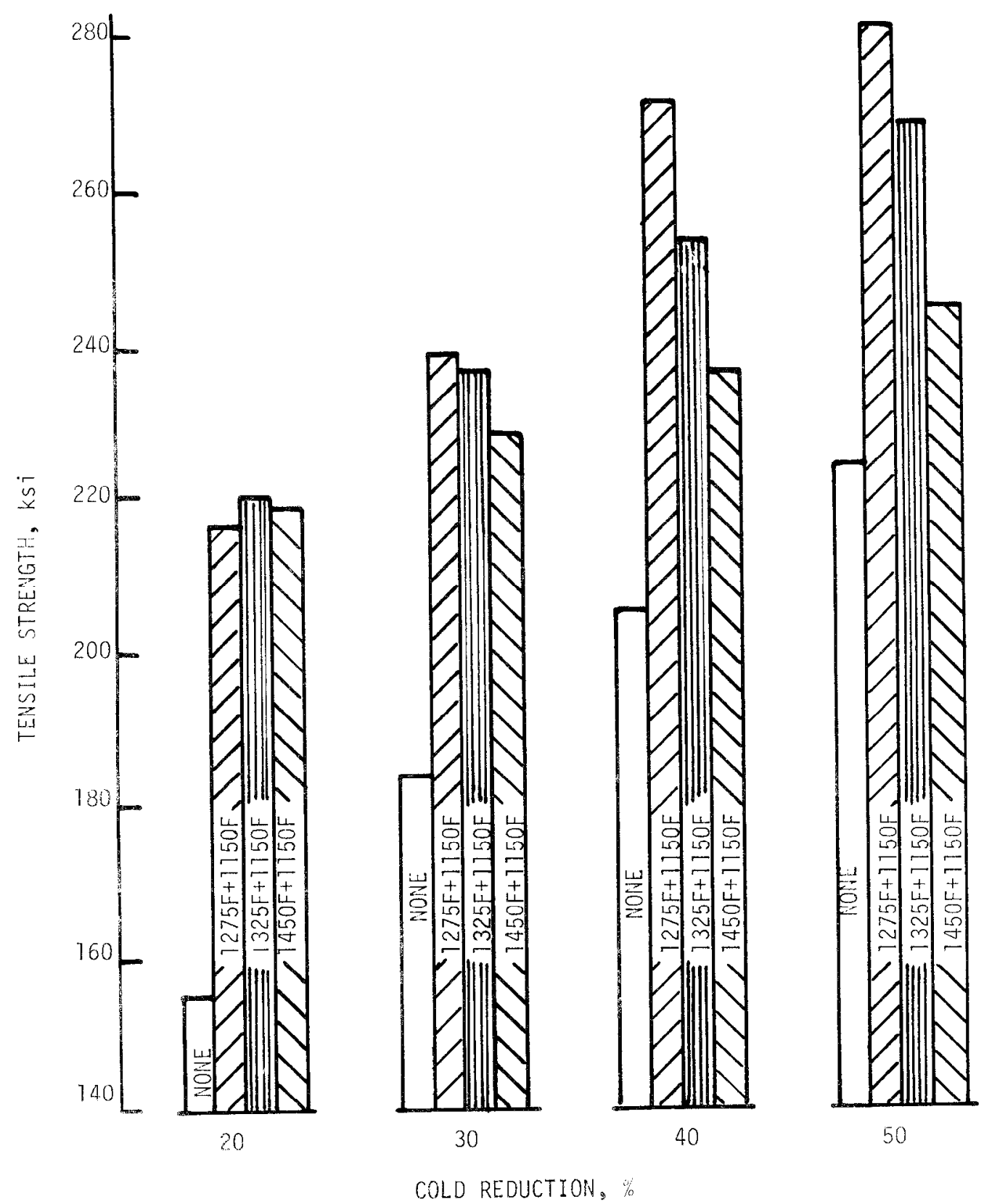

FIGURE 8. EFFECT OF AGING CYCLE ON TENSILE STRENGTH OF .875" ROUND BARS. $1750{ }^{\circ}$ PRIOR SOLUTION TREATMENT. 

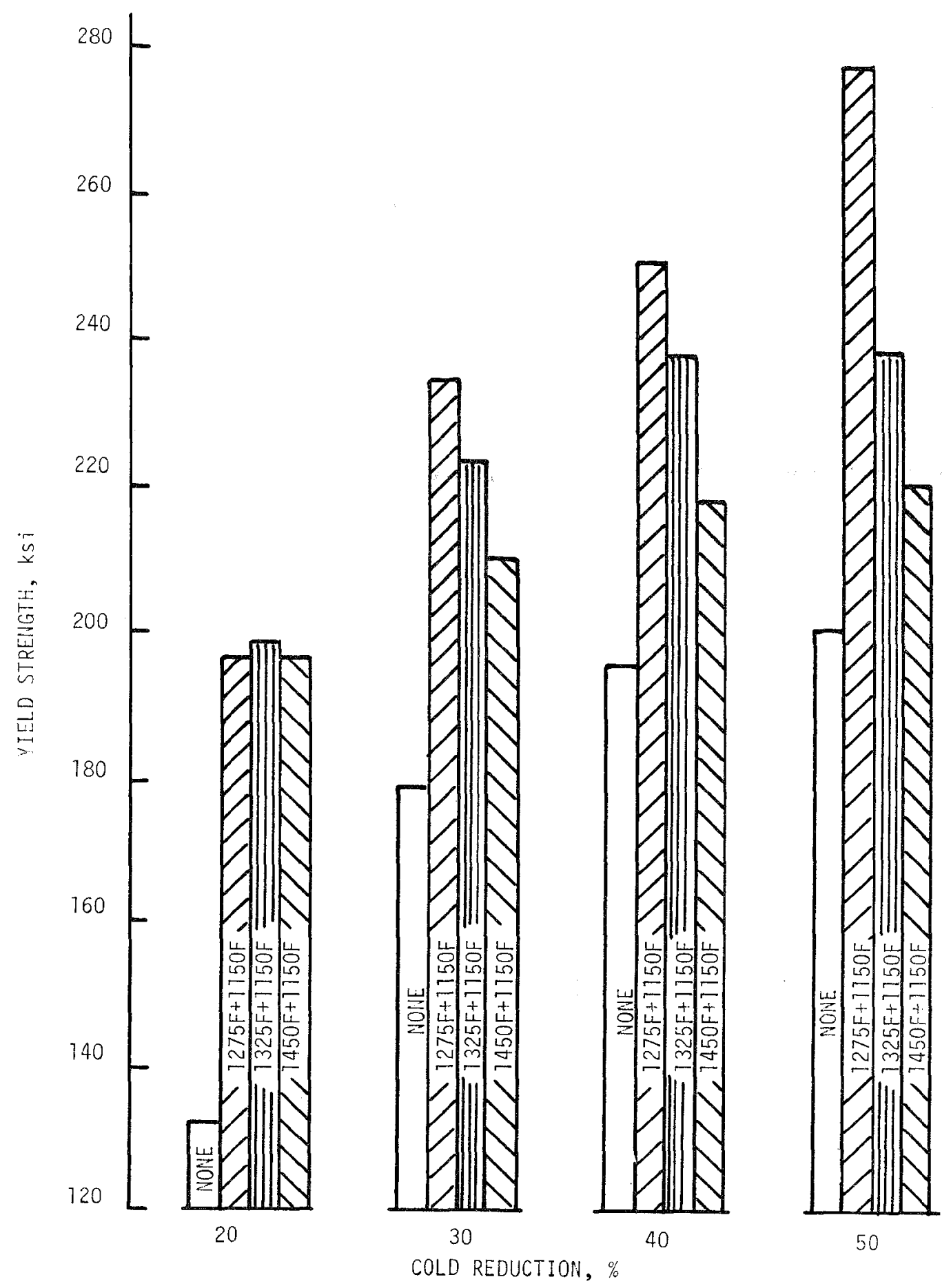

FIGURE 9. EFFECT OF AGING CYCLE ON YIELD STRENGTH OF .875" ROUND BARS. $1750 F$ PRIOR SOLUTION TREATMENT. 


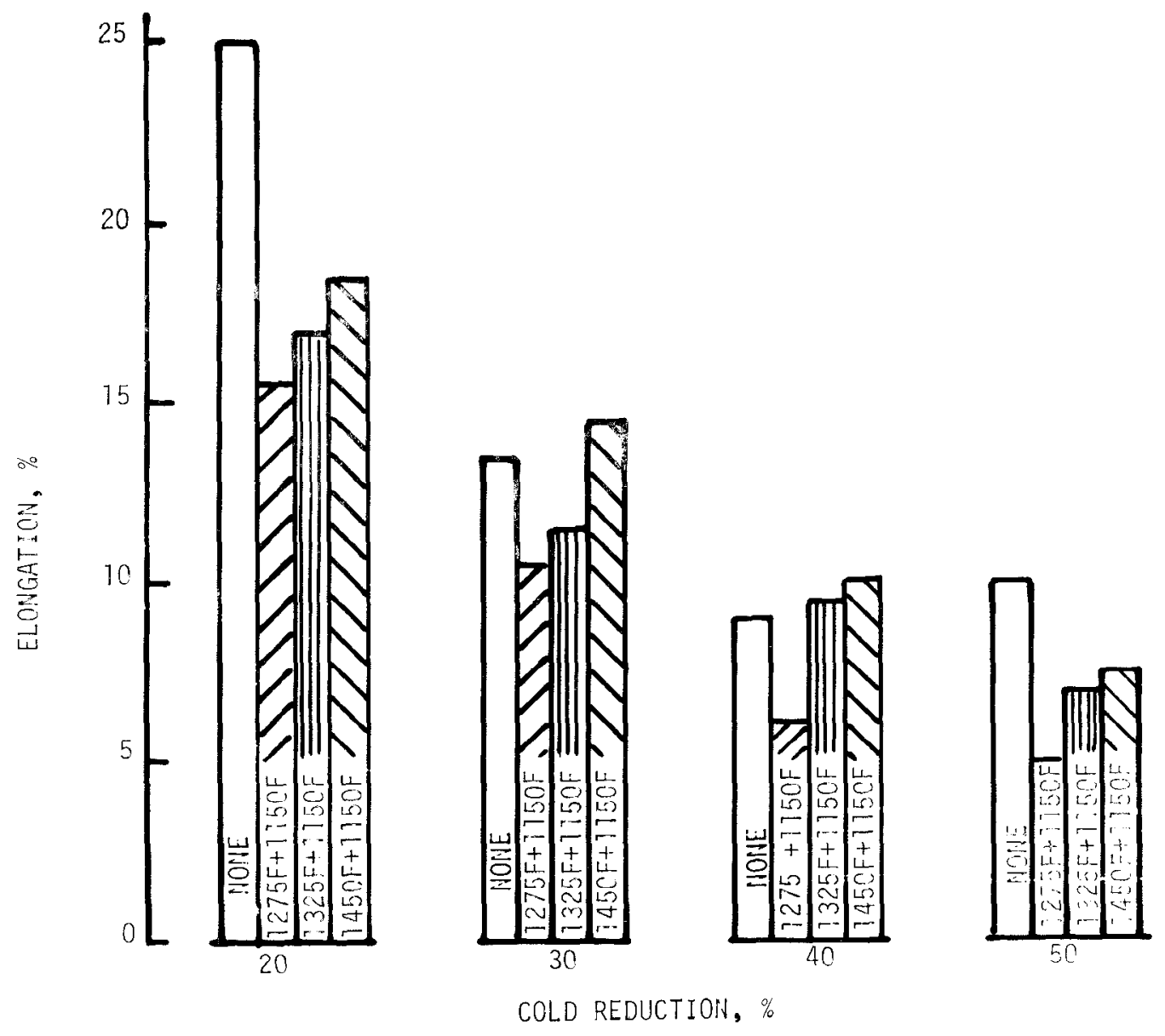

FIGURE 10. EFFECT OF AGING CYCLE ON TENSILE ELONGATION OF .875" ROUND RAR $1750 F$ PRIOR SOLUTION TREATMENTT. 


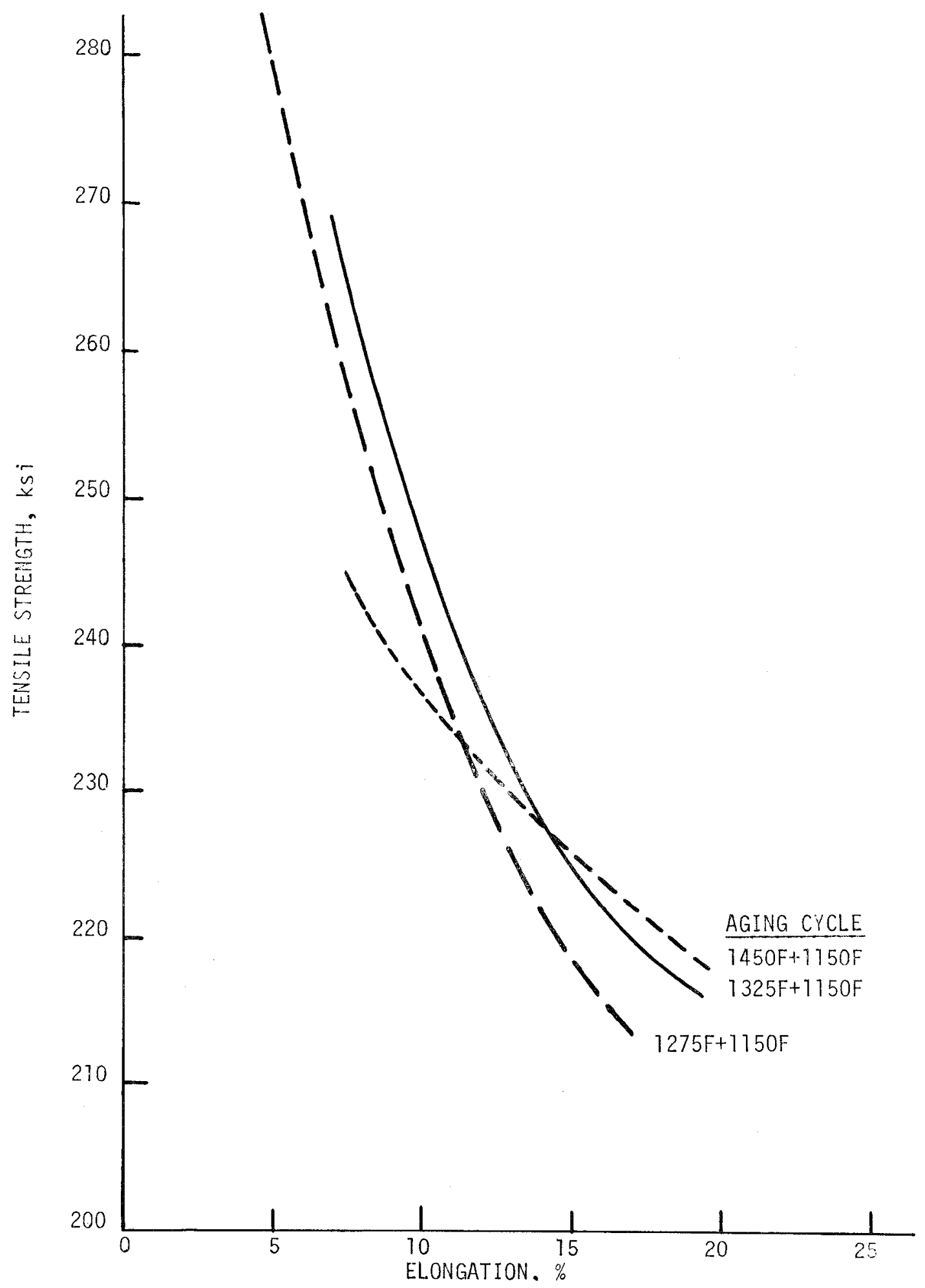

FIGURE 11. TENSILE STRENGTH AND ELONGATION OF ALL .875" ROUND BARS. 1750F PRIOR SOLUTION TREATMENT. 


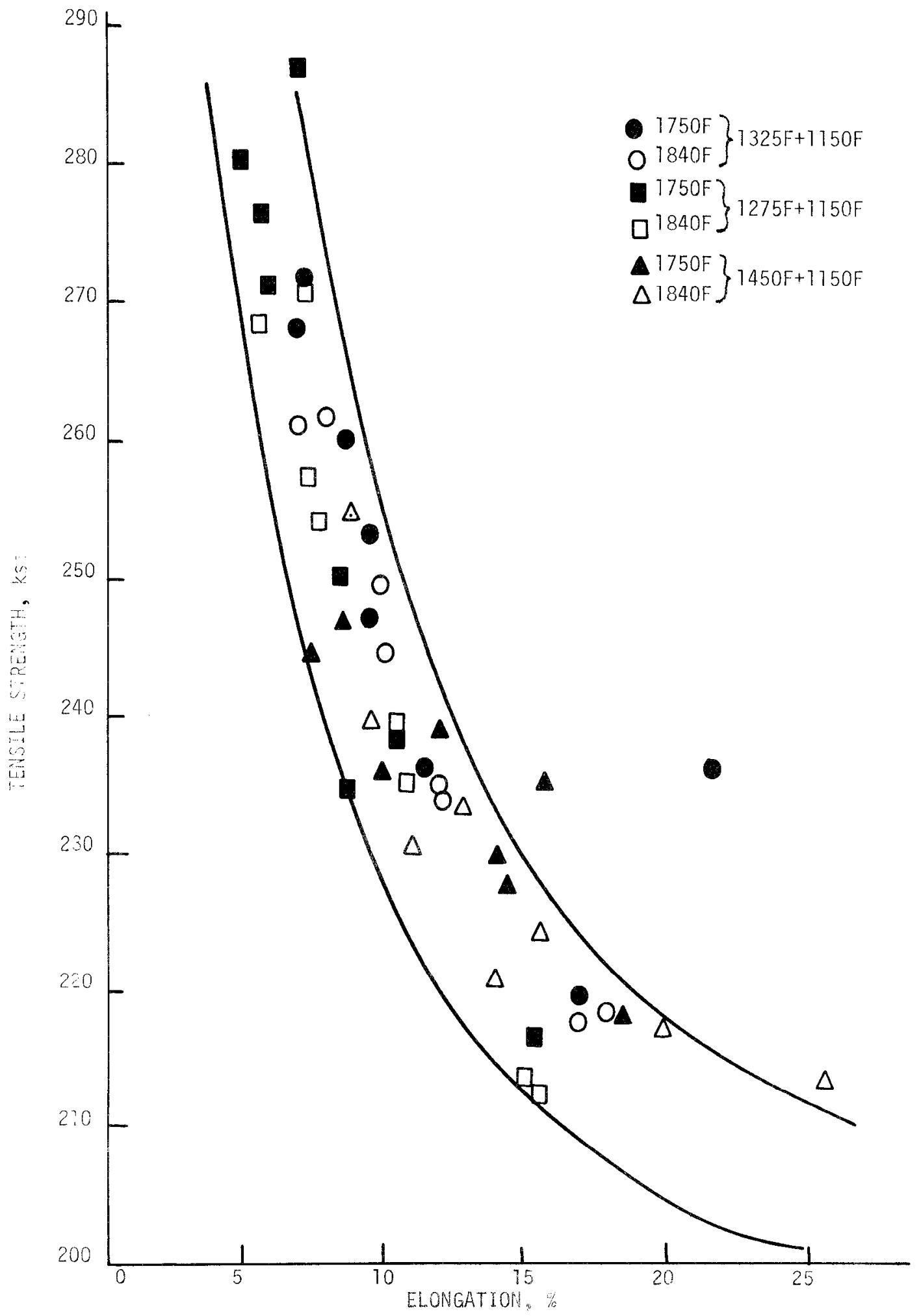

FIGURE 12. TENSILE STRENGTH ANI ELONGATION OF ALL SPECIMENS. 


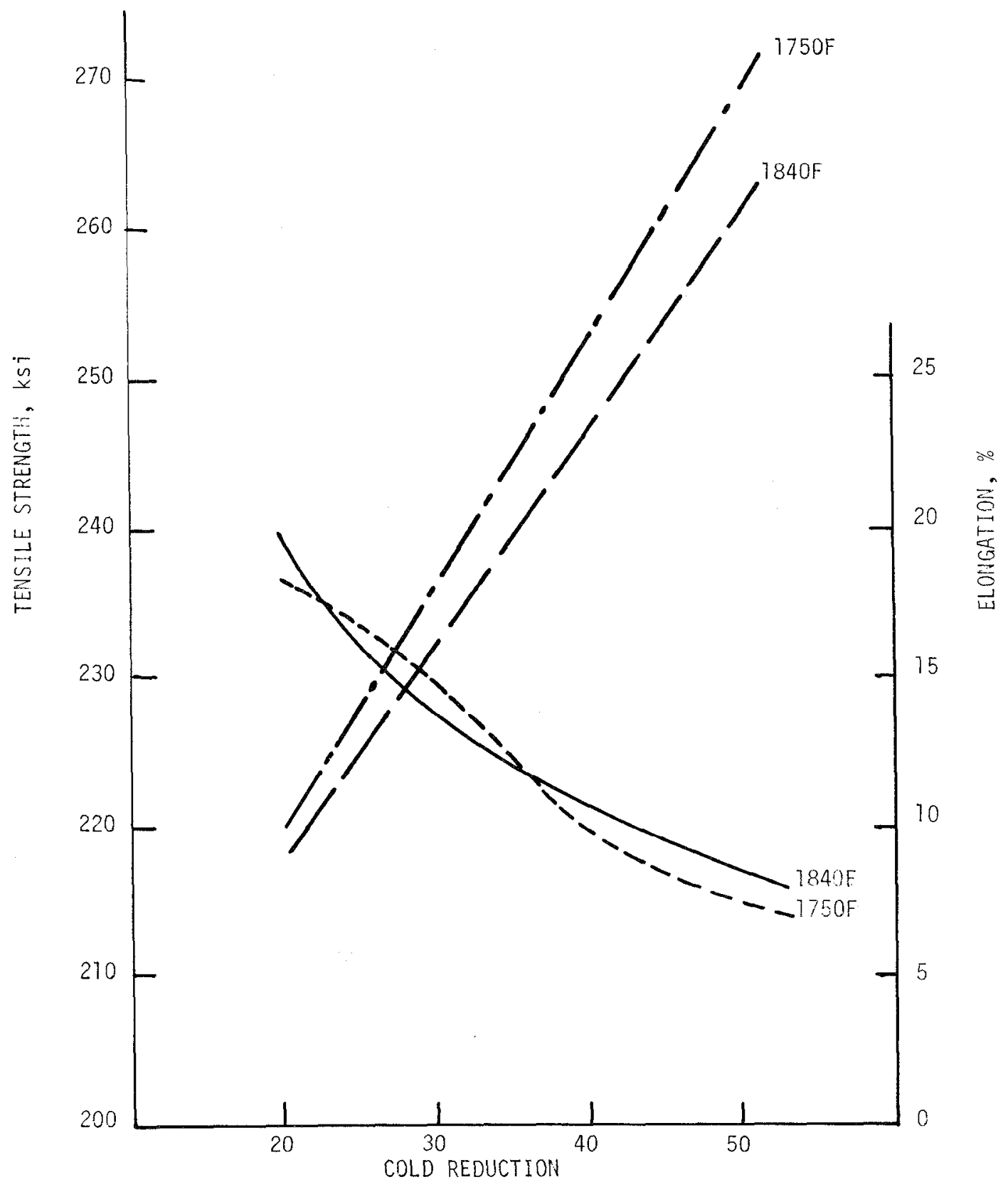

FIGURE 13. [FFECT OF PRIOR SOLUTIONING TEMTERATURE OII TENSILE STRENGTH AND TENSILE ELONGATION OF .875" ROUND BARS AFTER 1325F+1150F AGE. 


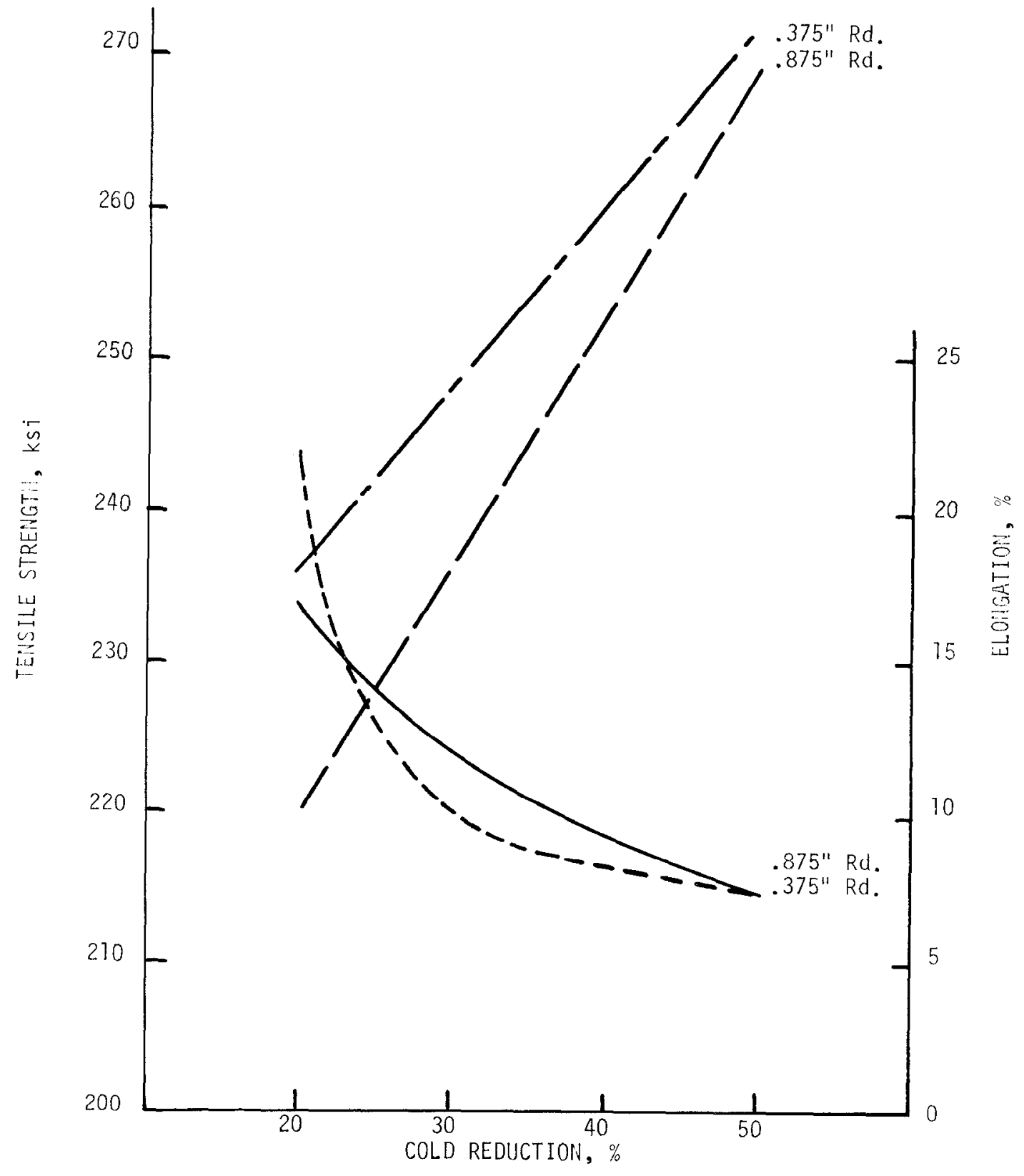

FIGURE 14. EFFECT OF BAR SIZE ON TENSILE STRENGTH AND TENSILE ELONGATION OF BARS AFTER 1325F+1150F AGE. 1750F PRIOR SOLUTION. 

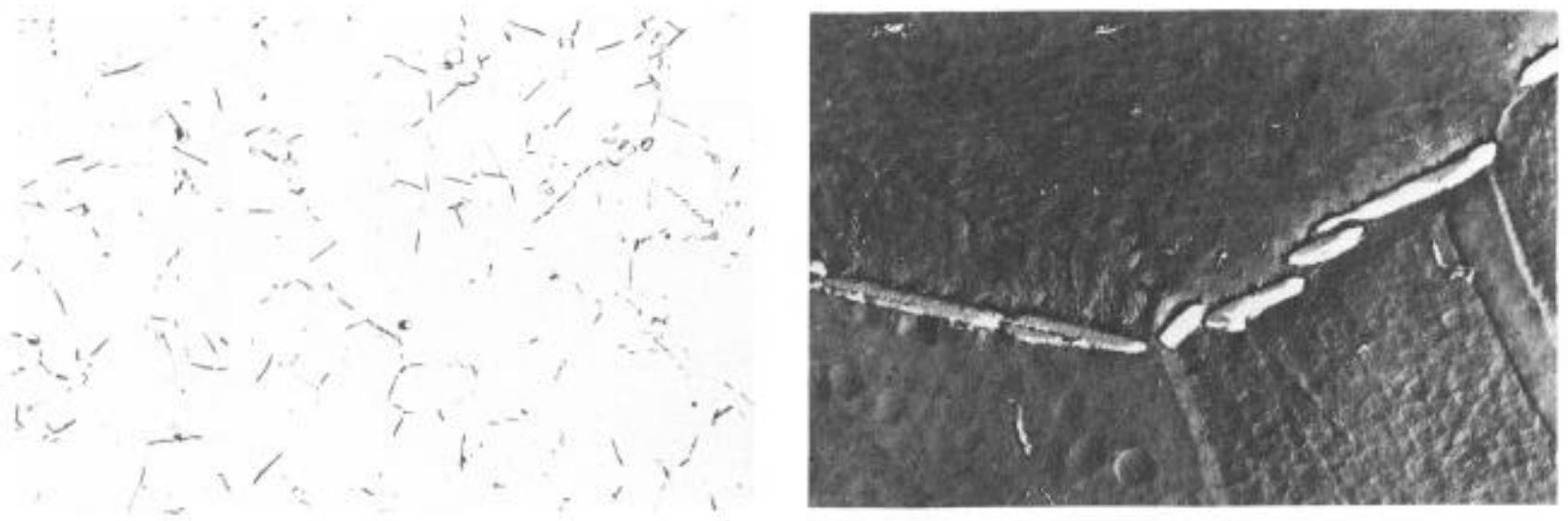

1750 F SHT - $20 \%$ REDUCTION

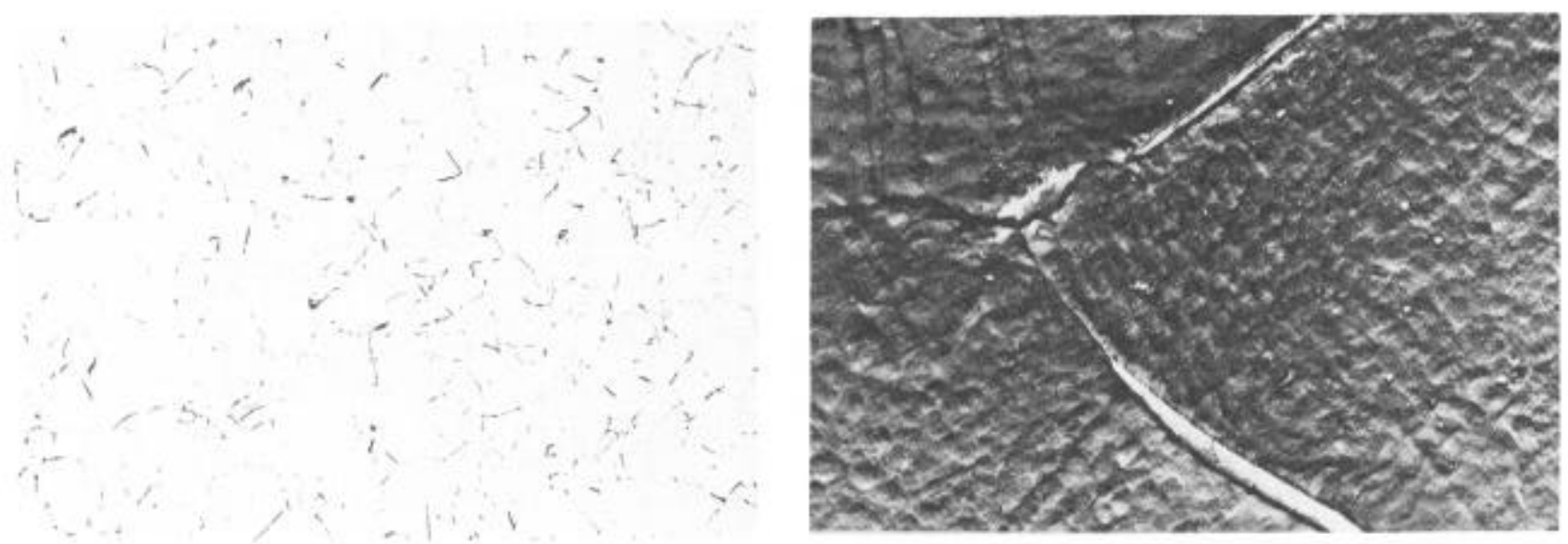

1750 F SHT - $50 \%$ REDUCTION
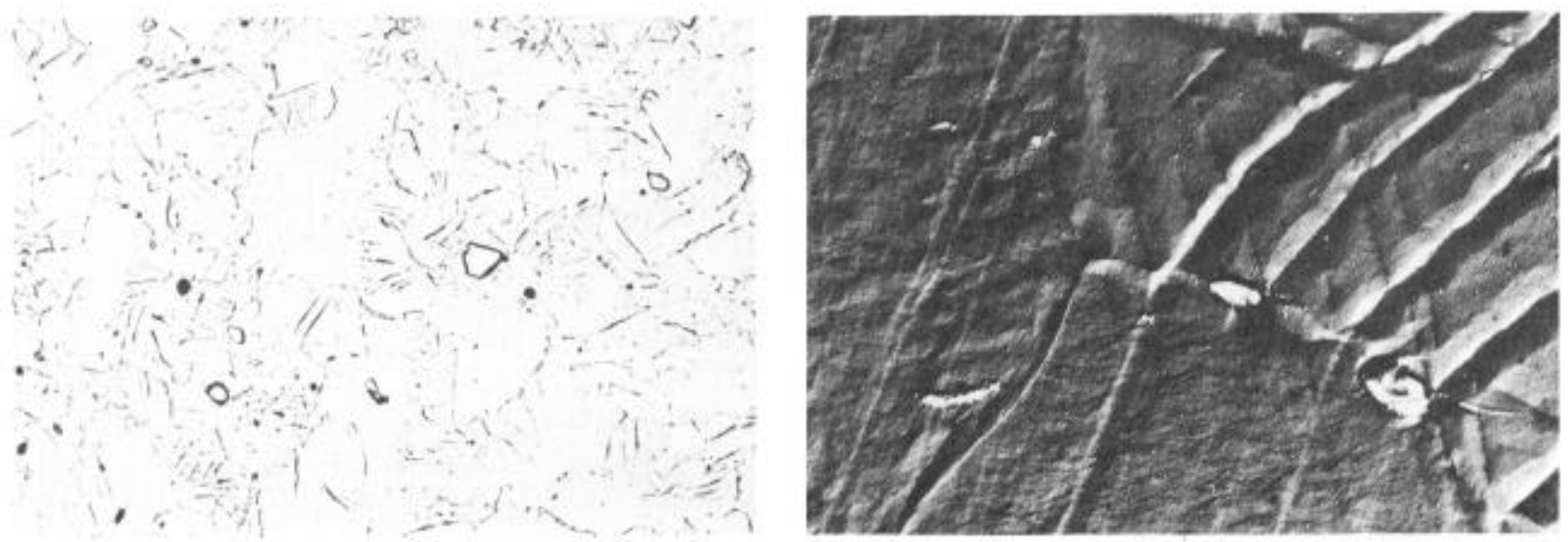

$1840 F$ SHT $-50 \%$ REDUCTION

FIGURE 15. MICROSTRUCTURES TYPICAL OF SPECIMENS IN COLD-REDUCED CONDITION. (.875" RD. BARS)

LIGHT MICROGRAPHS - 500X.

ELECTRON MICROGRAPHS - $9400 X$.

ETCHANT - FRYS. 

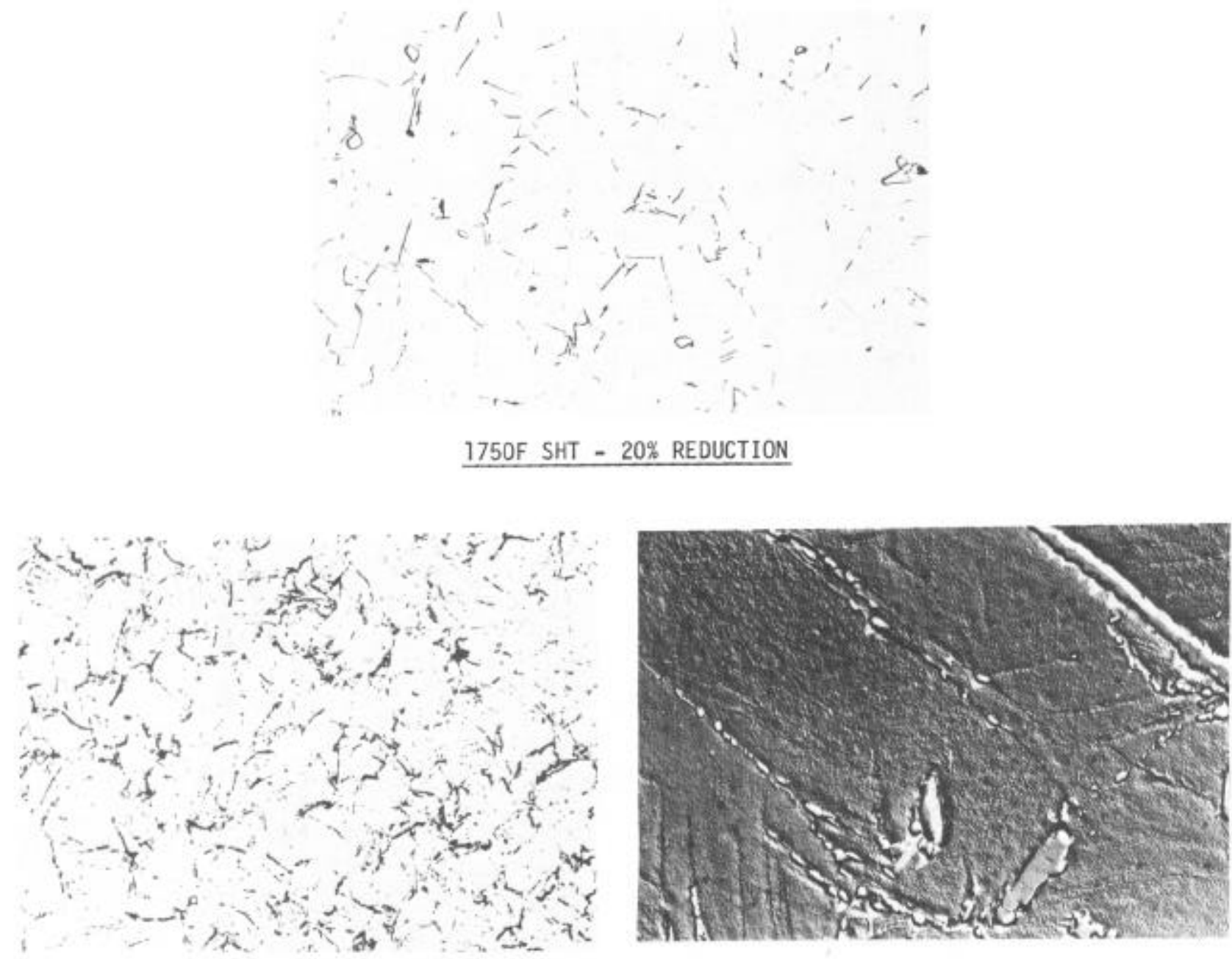

\section{$1750 F$ SHT - $50 \%$ REDUCTION}

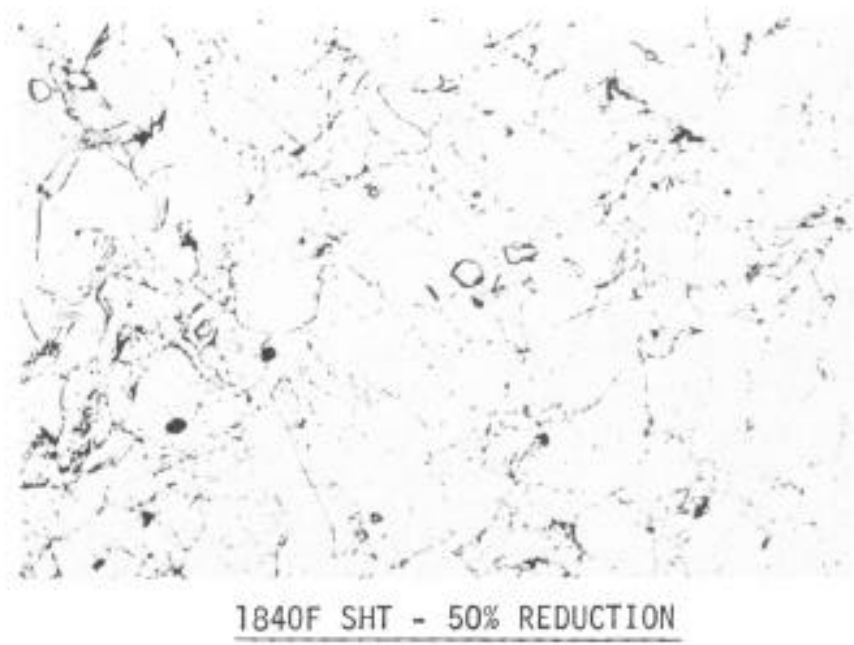

FIGURE 16. MICROSTRUCTURES TYPICAL OF SPECIMENS AFTER $1325 \mathrm{~F}+1150 \mathrm{~F}$ AGE.

(.875" RD. BARS)

LIGHT MICROGRAPHS - 500X.

ELECTRON MICROGRAPHS - $9400 x$. ETCHANT - FRYS. 

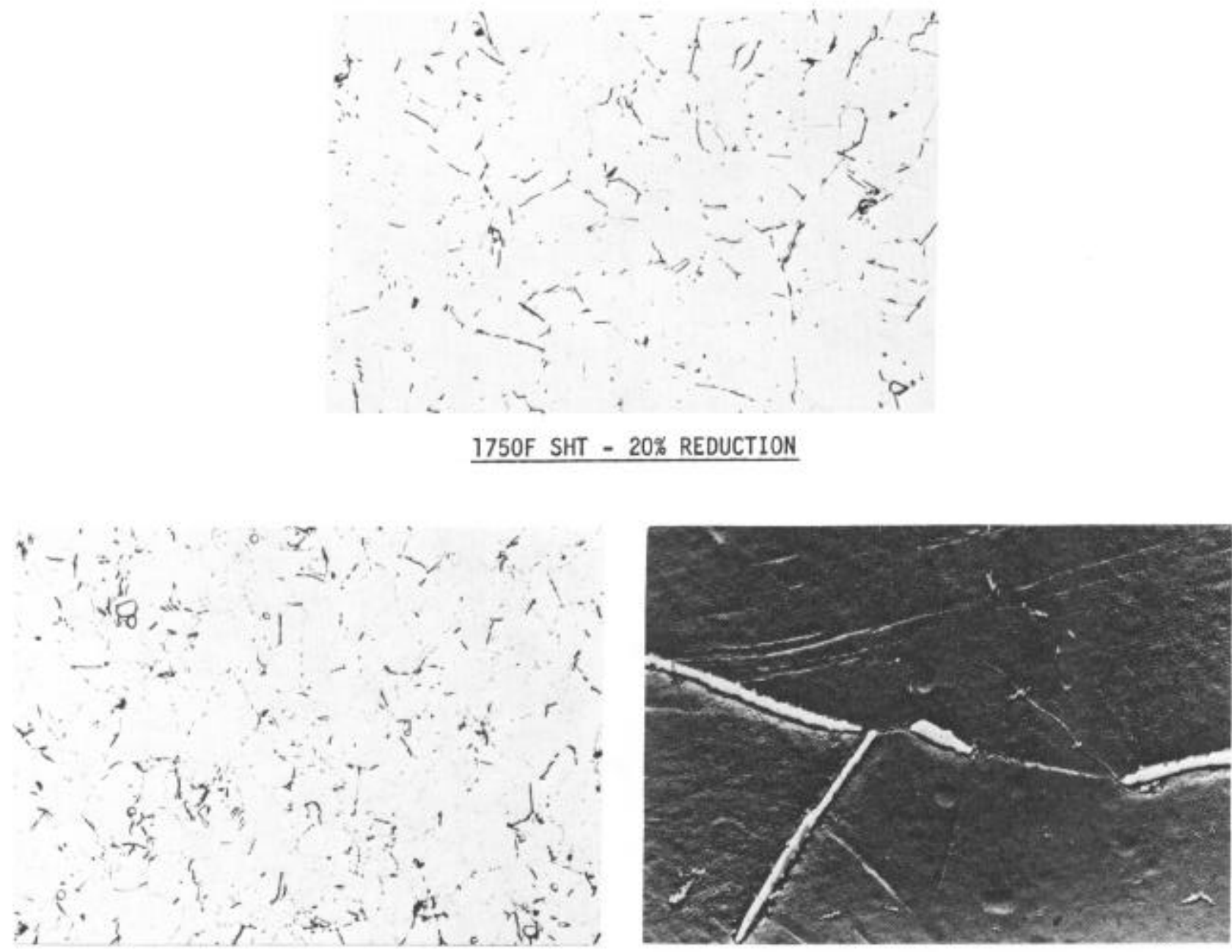

$1750 \mathrm{~F}$ SHT - 50\% REDUCTION

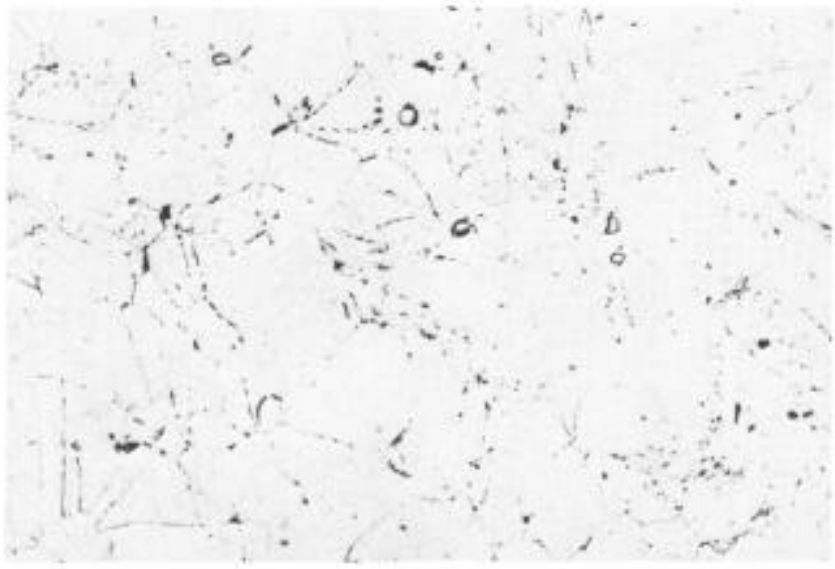

$1840 \mathrm{~F}$ SHT - 50\% REDUCTION

FIGURE 17. MICROSTRUCTURES TYPICAL OF SPECIMEN AFTER 1275F+1150F AGE. (.875" RD. BARS) 

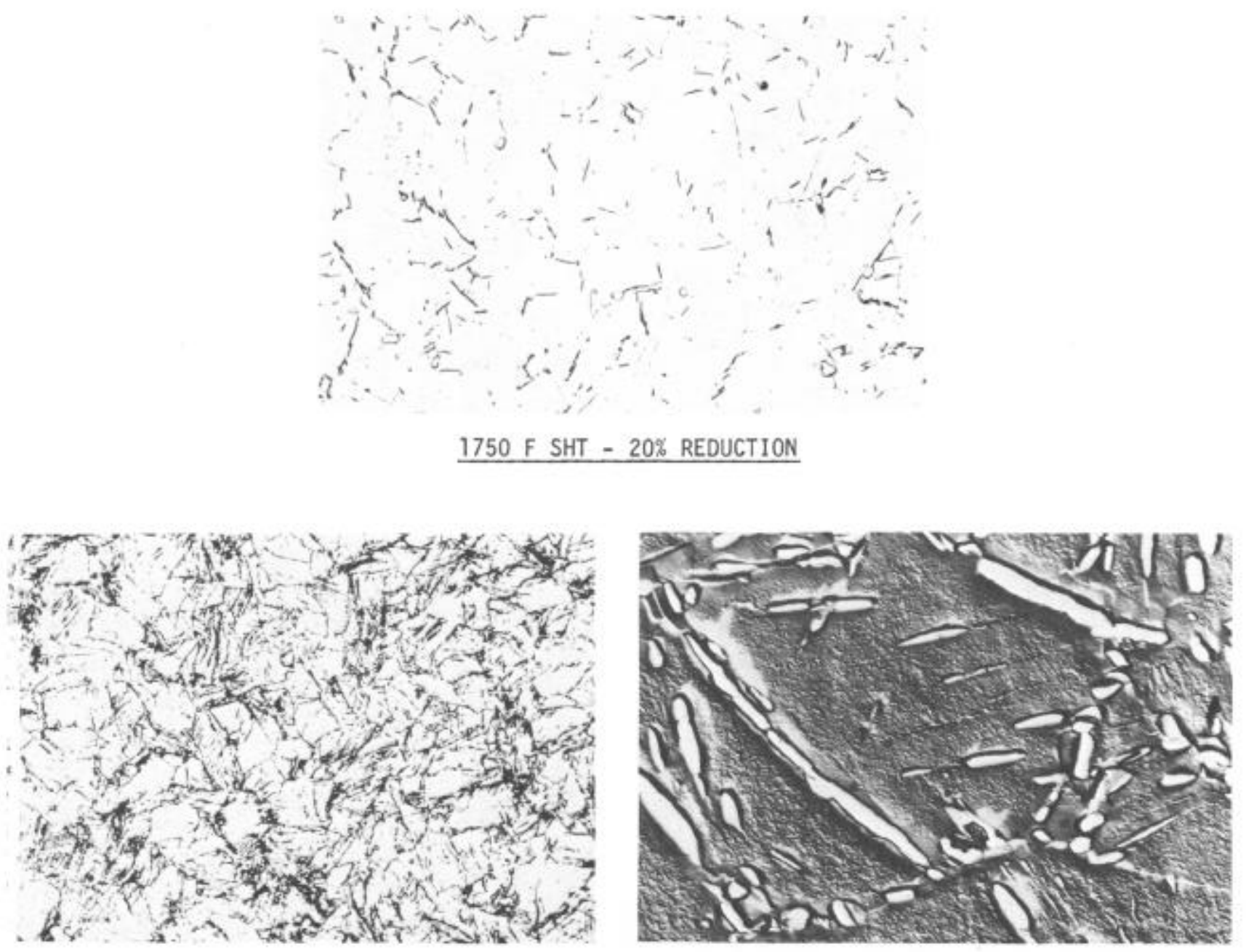

\section{F SHT - 50\% REDUCTION}

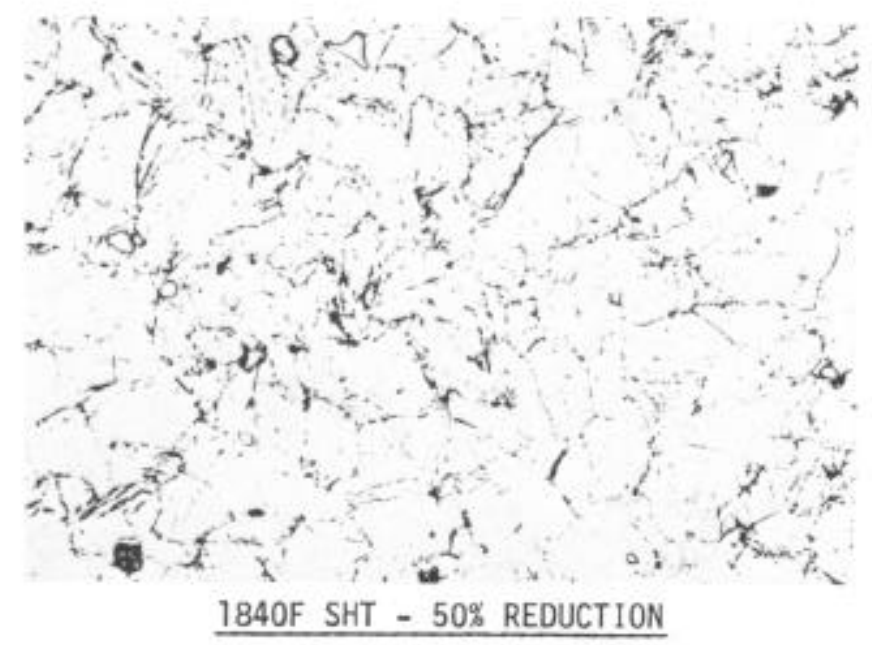

FIGURE 18. MICROSTRUCTURES TYPICAL OF SPECIMENS AFTER 1450F+1150F AGE. (.875" RD. BARS)

LIGHT MICROGRAPHS - 500X. ELECTRON MICROGRAPHS - $9400 \mathrm{X}$. ETCHANT - FRYS. 